\title{
The use of machine learning algorithms to design a generalized simplified denitrification model
}

\author{
F. Oehler, J. C. Rutherford, and G. Coco \\ National Institute of Water \& Atmospheric Research, P.O. Box 11115, Hamilton, New Zealand
}

Received: 11 February 2010 - Published in Biogeosciences Discuss.: 1 April 2010

Revised: 29 September 2010 - Accepted: 7 October 2010 - Published: 27 October 2010

\begin{abstract}
We propose to use machine learning (ML) algorithms to design a simplified denitrification model. Boosted regression trees (BRT) and artificial neural networks (ANN) were used to analyse the relationships and the relative influences of different input variables towards total denitrification, and an ANN was designed as a simplified model to simulate total nitrogen emissions from the denitrification process. To calibrate the BRT and ANN models and test this method, we used a database obtained collating datasets from the literature. We used bootstrapping to compute confidence intervals for the calibration and validation process. Both ML algorithms clearly outperformed a commonly used simplified model of nitrogen emissions, NEMIS, which is based on denitrification potential, temperature, soil water content and nitrate concentration. The ML models used soil organic matter $\%$ in place of a denitrification potential and $\mathrm{pH}$ as a fifth input variable. The BRT analysis reaffirms the importance of temperature, soil water content and nitrate concentration. Generalization, although limited to the data space of the database used to build the ML models, could be improved if $\mathrm{pH}$ is used to differentiate between soil types. Further improvements in model performance and generalization could be achieved by adding more data.
\end{abstract}

\section{Introduction}

The increase of agricultural nitrogen $(\mathrm{N})$ inputs favors the emission of nitrous oxide $\left(\mathrm{N}_{2} \mathrm{O}\right)$ through nitrification and denitrification. $\mathrm{N}_{2} \mathrm{O}$ is a well-known greenhouse gas (IPCC, 2006) involved in the ozone layer destruction (Cicerone, 1987) and soils are the main source of atmospheric $\mathrm{N}_{2} \mathrm{O}$ (Mosier and Kroeze, 2000). Indirect emissions of $\mathrm{N}$ gasses

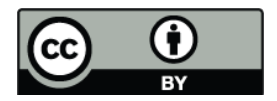

Correspondence to: F. Oehler (f.oehler@niwa.co.nz) (i.e., occurring after the applied nitrogen has been transformed or transferred out of the field) are still a major source of uncertainty despite their role on climate change (Crutzen et al., 2007; Mosier and Kroeze, 2000; Nevison, 2000).

Heterotrophic denitrification is the biological reduction of nitrate $\left(\mathrm{NO}_{3}^{-}\right)$or nitrite $\left(\mathrm{NO}_{2}^{-}\right)$into $\mathrm{N}_{2} \mathrm{O}$ and di-nitrogen $\left(\mathrm{N}_{2}\right)$ in absence of oxygen $\left(\mathrm{O}_{2}\right)$. The process is influenced by many factors, is highly variable over space and time, and is thus difficult to assess at the catchment level. The difference between annual nitrogen flow measured at the catchment outlet and the nitrogen surplus do not provide a reliable estimate of the denitrification at the catchment scale, because of temporary storage processes of nitrogen in the soil, vadose zone or groundwater (Basset-Mens et al., 2006; Molenat and Gascuel-Odoux, 2002; Ruiz et al., 2002). Furthermore, losses by gaseous emission through denitrification are not evenly distributed over the catchment area since they are particularly higher in the riparian zone (Fisher and Acreman, 2004; Haag and Kaupenjohann, 2001; Martin et al., 1999; Oehler et al., 2007; Sebilo et al., 2003). As a result, it is still problematic to up-scale measured emissions to a larger, landscape scale which is the most relevant to assess the impact of agriculture practices and their management.

Models can be used to take into account these processes and the spatial and temporal variability of the driving factors. Many models integrate a more or less complex denitrification module (e.g. GLEAMS (Knisel, 1993), DNDC (Li et al., 1992), SWAT (Arnold and Fohrer, 2005), TNT2 (Beaujouan et al., 2001)) to simulate $\mathrm{NO}_{3}^{-}$fluxes at the agricultural field or catchment scale. These models are often coupled to socioeconomic models to provide an integrated $\mathrm{N}$ management tool (Leip et al., 2008; Turpin et al., 2005). Different approaches have been developed for denitrification modelling. These approaches range from (1) simplified process models (e.g. NEMIS, Henault and Germon, 2000), (2) to soil structural models (e.g. Vinten et al., 1996), and (3) to microbial growth models (e.g. DNDC).

Published by Copernicus Publications on behalf of the European Geosciences Union. 
The accuracy of measurement techniques still needs to be improved especially to assess long term emissions, and this is particularly the case for upland terrestrial areas (Groffman et al., 2006). Our long term goal is developing a model of denitrification at the catchment scale that also addresses the significant emissions from upland areas (Oehler et al., 2007). To achieve this aim, we turned towards simplified modelling approaches also because (1) mechanistic models are developed and validated for homogeneous and simple medium, which is not necessarily appropriate at the catchment scale (Beven, 1993), (2) either the accuracy of measured emissions is poor and/or sampling is too scarce (Groffman et al., 2006), (3) simplified models need inputs that can be obtained either from relatively simple field measurements or directly from simulation models.

Simplified models have already been used in many studies and, for example, Heinen (2006b) found as many as 59 simplified models in the literature. He also analyzed the performance of the simplified model NEMIS on an extended data set (Heinen, 2006a). Following the same procedure as Heinen (2006a), NEMIS was also calibrated on another large data set (Oehler et al., 2009). Because of either measurements or modelling shortcomings, results were not fully satisfactory for a generalized use at the catchment scale. Moreover, there is a need to simulate also $\mathrm{N}_{2} \mathrm{O}$ emissions from denitrification at the catchment scale, especially as stakeholders are looking toward the use of wetlands as nitrogen attenuation tools. Finally, it is worth reminding that in simplified approaches the global $\mathrm{N}$ emissions are a key parameter to estimate $\mathrm{N}_{2} \mathrm{O}$ (using the $\mathrm{N}_{2} \mathrm{O} / \mathrm{N}_{2}$ ratio, Henault et al., 2005; Lehuger et al., 2009) and so their estimate needs to be more robust and accurate.

Simplified models can be developed using a data-driven approach and so using a broad family of algorithms loosely defined in the literature as "machine learning" (ML). The core objective of a Machine Learning algorithm is to generalize from its experience, i.e. to provide a model that captures the overall characteristics and interactions of the dataset it has been trained on (Alpaydin, 2004). These by-design generalized models are however limited in their application by the extent of the gradients present in the dataset used to construct them. The degree of generalization, also called "generalization error" or "performance" is often evaluated using a cross or independent validation process (Bousquet and Elisseeff, 2002; Elith et al., 2008; Hagan et al., 1996). These techniques provide an assessment of how well the generalized models behave on unseen data inside the range of the training dataset.

Since the "universal approximator demonstration" at the end of the 1980's (Cybenko, 1989; Hornik et al., 1989; Irie and Miyake, 1988), artificial neural networks (ANN) have probably become the most typical machine learning algorithm and have been used in many different fields like physics, chemistry, medicine, ecology and hydrology (Cote et al., 1995; Faraggi and Simon, 1995; Kralisch et al., 2003;
Lek et al., 1999; Lischeid, 2001; Smits et al., 1992; Suen and Eheart, 2003; Telszewski et al., 2009). Artificial Neural Networks have been widely used to model complex nonlinear relationships, particularly when the functional form of the relations between the variables involved is unknown.

Boosted Regression Trees (BRT) is a relatively new ML algorithm (partly originating from Schapire, 2003) characterized by strong predictive performance and that can give powerful insights of the variable relationships (Elith et al., 2008). However BRT are complex models in their representation (from a few hundred to few thousands of trees), and can be difficult to export from the ML environment to a separate and independent model. BRT do not entirely fit into the "simplified models", but they can efficiently describe the relationship between input variables and a system response.

We used the BRT approach to specifiquely study the variable relative influences and relationship and to help selecting the most relevant variables. Then an ANN was provided, taking advantage of its relative simplicity and portability with a (hopefully) small tradeoff in performance.

In order to test this method of designing a simplified model based on ANN to simulate $\mathrm{N}$ emissions from the denitrification process at the field scale, we:

- assembled a database from literature datasets;

- analysed the variable relationships and the relative influences of input variables toward total denitrification.

- carefully assessed the generalization error of the ML approaches and compared them with NEMIS;

- explored the sensitivity of simulated denitrification rates to input factor variations.

\section{Methods}

\subsection{Database and input factors}

To calibrate (train) the BRT and ANN models and test the method, a large enough database is needed. The dasabase was built with datasets easily extractable from the literature (Cosandey et al., 2003; Henault and Germon, 2000; Luo et al., 1999; Oehler et al., 2007; Ryden, 1983; Zaman and Nguyen, 2010). Denitrification rate $(D a)$ rates were measured using the acetylene $\left(\mathrm{C}_{2} \mathrm{H}_{2}\right)$ blockage technique (Ryden et al., 1987; Yoshinari et al., 1977). The soil denitrifying potential was either a long term (days, termed Denitrification Potential (LDP) as in Henault and Germon, 2000) or a short term (hours, termed Denitrifying Enzyme Activity (DEA) as in Cosandey et al., 2003; Luo et al., 1999; Oehler et al., 2007; Zaman and Nguyen, 2010) measure. The main differences in measurement techniques are summarized in Table 1. For the dataset of Luo et al. (1999), soil temperature $(T)$ was estimated from national statistics. All 
Table 1. The different measurement methods of $D a$ and DEA in the database.

\begin{tabular}{|c|c|c|c|c|}
\hline Source & Measure & Method variant & Incubation time & $\begin{array}{l}\text { Incubation } \\
\text { temperature }\end{array}$ \\
\hline \multirow[t]{2}{*}{ Cosandey et al. (2003) } & DEA & $\begin{array}{l}\text { Smith and Tiedje (1979), } \\
\text { flasks, mixed }\end{array}$ & $4 \mathrm{~h}$ & $20^{\circ} \mathrm{C}$ \\
\hline & $D a$ & $\begin{array}{l}\text { Yoshinari and Knowles (1976), } \\
\text { flasks, disturbed soil samples }\end{array}$ & $4 \mathrm{~h}$ & $20^{\circ} \mathrm{C}$ \\
\hline Henault and Germon (2000) & $D a$ & $\begin{array}{l}\text { Adaptation of Tiedje et al. (1989), } \\
\text { Soil cores, undisturbed }\end{array}$ & $3 \mathrm{~h}$ to days & $20^{\circ} \mathrm{C}$ \\
\hline \multirow[t]{2}{*}{ Luo et al. (1999) } & DEA & Luo et al. (1996), flasks, mixed & $5 \mathrm{~h}$ & \multirow{2}{*}{$\begin{array}{l}20^{\circ} \mathrm{C} \\
\text { daily soil } \\
\text { temperature } \\
\text { variation }\end{array}$} \\
\hline & $D a$ & $\begin{array}{l}\text { Ryden et al. (1987), } \\
\text { Soil cores, slightly disturbed }\end{array}$ & $24 \mathrm{~h}$ & \\
\hline \multirow[t]{2}{*}{ Oehler et al. (2007) } & DEA & Luo et al. (1996), flasks, mixed & $5 \mathrm{~h} 30 \mathrm{~min}$ & \multirow{2}{*}{$\begin{array}{l}20^{\circ} \mathrm{C} \\
\text { daily soil } \\
\text { temperature } \\
\text { variation }\end{array}$} \\
\hline & $D a$ & $\begin{array}{l}\text { Adaptation of Jarvis et al. (2001), } \\
\text { Soil cores, undisturbed }\end{array}$ & $24 \mathrm{~h}$ & \\
\hline Ryden (1983) & $D a$ & $\begin{array}{l}\text { Ryden and Dawson (1982), direct } \\
\text { on-site measurement, undisturbed }\end{array}$ & $3.5 \mathrm{~h}$ & $\begin{array}{l}\text { actual soil } \\
\text { temperature }\end{array}$ \\
\hline \multirow[t]{2}{*}{ Zaman and Nguyen (2010) } & DEA & Tiedje (1982), flasks, mixed & $7 \mathrm{~h}$ & \multirow{2}{*}{$\begin{array}{l}20^{\circ} \mathrm{C} \\
\text { daily soil } \\
\text { temperature } \\
\text { variation }\end{array}$} \\
\hline & $D a$ & $\begin{array}{l}\text { Adaptation of Tiedje et al. (1989), } \\
\text { Soil cores, undisturbed }\end{array}$ & $24 \mathrm{~h}$ & \\
\hline
\end{tabular}

the studies were carried out in temperate regions (France, Switzerland, south-east of England and New Zealand) and $34 \%$ of the measurements were in riparian or wetland areas. Our final database has 536 records: 58 from Cosandey et al. (2003); 39 from Henault and Germon (2000); 99 from Luo et al. (1999); 253 from Oehler et al. (2007); 46 from Ryden (1983) and 41 from Zaman and Nguyen (2010). Soil types included: cultivated and uncultivated silt loam and silty clay loam soils with OM 4-7\% and pH 5-6.5; cultivated silt loam with OM $1 \%$ and $\mathrm{pH} 7.1$; grazed riparian grasslands on silty clay and silty sand soils with OM 2.4-12.2\% and pH 6.8-8; and pasture on silt loam with $\mathrm{OM} 6 \%$ and $\mathrm{pH}$. All the $D a$ measurements were done using a static chamber technique. The main denitrification measurements issues with the $\mathrm{C}_{2} \mathrm{H}_{2}$ blockage technique are:

- the diffusion of $\mathrm{C}_{2} \mathrm{H}_{2}$ into the soil;

- $\mathrm{C}_{2} \mathrm{H}_{2}$ can be used as a carbon source by micro-organism after a long time;

- $\mathrm{C}_{2} \mathrm{H}_{2}$ inhibits also the mineralisation, hence limiting its applicability to moderate to high $\left[\mathrm{NO}_{3}^{-}\right]$;

- the diffusion of $\mathrm{O}_{2}$ into the soil samples if they are disturbed;
- low gas emission dynamic compared to the sensor sensitivity, compensated by the length of incubation time;

- soils are heterogeneous substrates.

All of this can lead to a large measurement variability, especially for low-drainage soils (Groffman et al., 2006). Henault and Germon (2000) and Cosandey et al. (2003) measurements may be the less variable (i.e. all the measurements at $20^{\circ} \mathrm{C}$ ). The DEA measurement methods are similar in their adding of substrate quantities, mixing procedures and incubation time.

Figure 1 shows the distribution of the response ( $D a$ rates) and independent variables $\left(\mathrm{NO}_{3}^{-}, T\right.$, WFPS, OM, $D b, \mathrm{SD}$ and $\mathrm{pH}$ ). $\mathrm{Da}$ and $\mathrm{NO}_{3}^{-}$show distributions with very long upper tails. Table 2 shows the univariate linear correlations (Pearson $r$ ) between variables. The $r$ values (notice $r^{2}$ will be even smaller) show that we are not in a simple case with one or two dominant factors and linear relationships. $D a$ is weakly correlated with WFPS, $T, \mathrm{pH}$ and OM, but $D a$ is not correlated with $\mathrm{NO}_{3}^{-}$. This does not mean that $\mathrm{NO}_{3}^{-}$is not involved in the denitrification process. First, $\mathrm{NO}_{3}^{-}$may have been present (mostly) in excess so that it did not limit Da. Also, $\mathrm{NO}_{3}^{-}$soil concentration may have been a poor indicator of the rate of supply of $\mathrm{NO}_{3}^{-}$(e.g., by advection, diffusion or nitrification, if its inhibition by $\mathrm{C}_{2} \mathrm{H}_{2}$ was not 

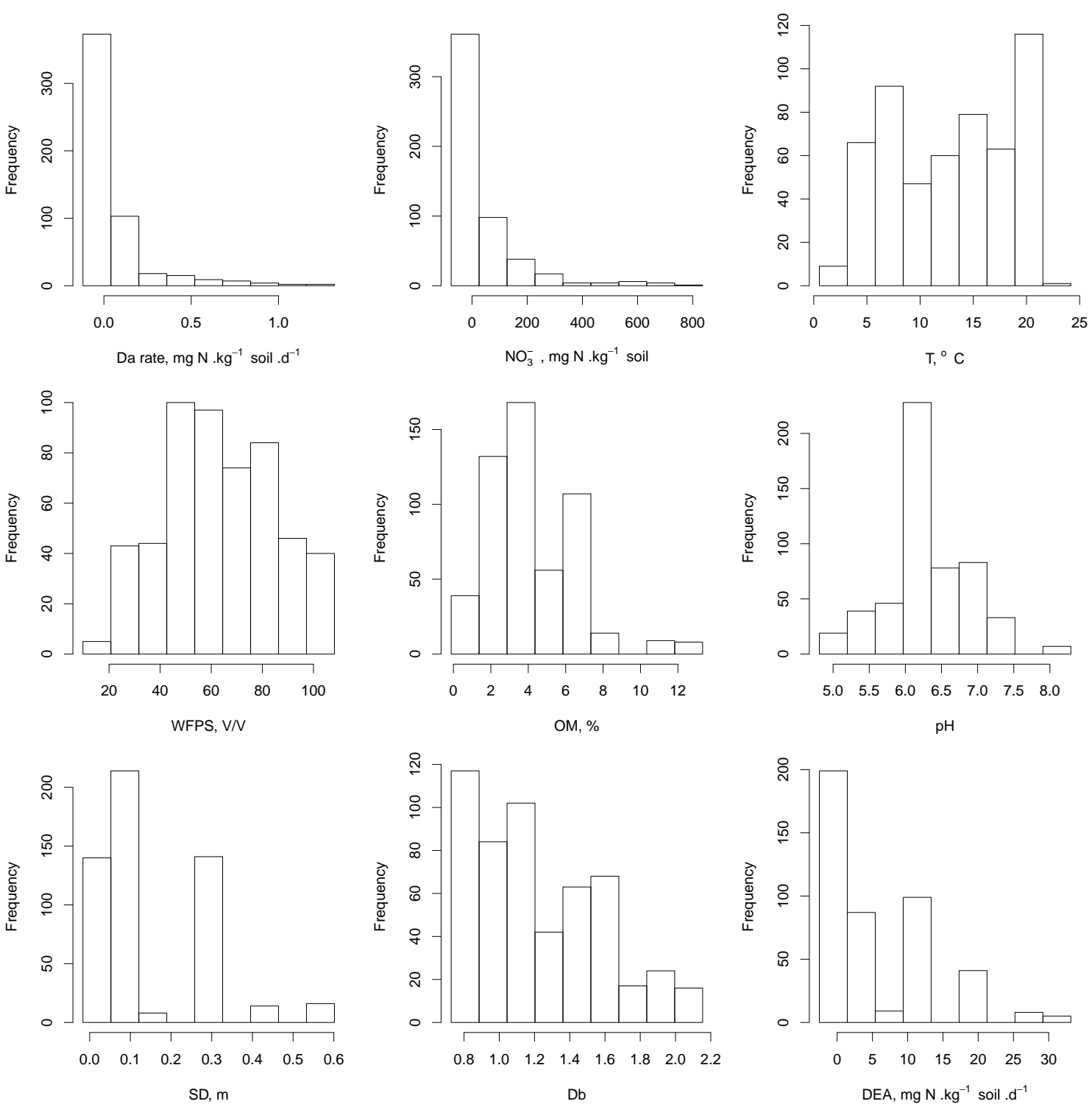

Fig. 1. Distribution of the variables in the database (536 records).

total) to denitrification micro sites, which is what determines $D a$. Second, the measurements from this collated dataset are far from genuinely and equally representing the different studied systems: there is a mixture of field (uncontrolled) and laboratory (some of the parameters are controlled or manipulated, like $T$ or $\left[\mathrm{NO}_{3}^{-}\right]$) measurements, with different sampling strategies and measurement technique variants (disturbed or undisturbed soil cores). There are also correlations between the input variables. Notably OM and $\mathrm{NO}_{3}^{-}$are weakly correlated $(r=0.26)$ as a result of a small number of high $\mathrm{NO}_{3}^{-}$/low OM points in Henault and Germon (2000) and low $\mathrm{NO}_{3}^{-}$/high OM points in Cosandey et al. (2003). $D b$ is weakly correlated with OM $(r=$ $-0.43), \mathrm{pH}(r=0.60)$ and SD $(r=0.60)$, and $\mathrm{pH}$ is weakly correlated with $T(r=0.39)$. This might be again the result of a sampling bias (e.g., the highest $D b$ and $\mathrm{pH}$ soils were measured at $20^{\circ} \mathrm{C}$ in Henault and Germon (2000) and Cosandey et al. (2003), and the soils with highest $D b$ were also those with the highest $\mathrm{pH}$ ). Besides conjectures, at this stage we can only suggest that the variation of $D a$ is due to more than one factor and probably in a non-linear way. Because this is a multi-variable non-linear problem, $r$ values, a measure of the strength of a linear relationship between two variables, can only be used to deduce that the dataset might be unbalanced ( $r$ values between known factors not close to zero), and therefore that some variable-space regions might not be equally represented.

A clustering (partitioning) of the dataset (without the DEA) has been done with the PAM k-medoid method 
Table 2. Pearson $r$ correlation coefficient. Statistical significance indicated by $*(p<0.05)$.

\begin{tabular}{|c|c|c|c|c|c|c|c|c|c|}
\hline Pearson $r$ & $D a$ & DEA & $\mathrm{NO}_{3}^{-}$ & WFPS & $T$ & $\mathrm{pH}$ & $\mathrm{OM}$ & $D b$ & SD \\
\hline$D a$ & - & 0.07 & $0.19^{*}$ & $0.29^{*}$ & $0.36^{*}$ & $0.29^{*}$ & $-0.26^{*}$ & $0.15^{*}$ & -0.09 \\
\hline DEA & & - & $-0.23^{*}$ & $0.16^{*}$ & 0.07 & $0.21^{*}$ & $0.60^{*}$ & $-0.41^{*}$ & $-0.44^{*}$ \\
\hline $\mathrm{NO}_{3}^{-}$ & & & - & $-0.16^{*}$ & $0.14^{*}$ & $0.12^{*}$ & $-0.26^{*}$ & $0.25^{*}$ & 0.06 \\
\hline WFPS & & & & - & 0.03 & $0.20^{*}$ & 0.10 & $0.21^{*}$ & $0.10^{*}$ \\
\hline$T$ & & & & & - & $0.39 *$ & 0.03 & $0.28^{*}$ & $0.22 *$ \\
\hline $\mathrm{pH}$ & & & & & & - & 0.03 & $0.55^{*}$ & $0.18^{*}$ \\
\hline $\mathrm{OM}$ & & & & & & & - & $-0.43^{*}$ & $-0.15^{*}$ \\
\hline$D b$ & & & & & & & & - & $0.60^{*}$ \\
\hline SD & & & & & & & & & - \\
\hline
\end{tabular}

(Kaufman and Rousseeuw, 2005). We used the cluster $\mathrm{R}$ package 1.13.1 (Maechler et al., 2005) and the optimal number of classes was chosen with the silhouette method (Rousseeuw, 1987). The best silhouette score was obtained with 3 clusters (score of 0.32 ), quickly dropping for more clusters ( 0.19 for 4 clusters). Table 3 shows the counts of records in each cluster per source, as well as the medoids (i.e. the "central" point of each cluster). Overall, the dataset cannot be very well clustered, and only a small fraction (21\%) of the records seems to be differentiated. The dataset from Cosandey et al. (2003) is different (cluster 2) because of the temperature and ph values (Table 3) while the data from Oehler et al. (2007) constitutes another separate cluster characterized by high to very high $\mathrm{NO}_{3}^{-}$in cluster 3 (Table 3). These results indicate that the dataset is not strongly heterogeneous (optimal number of clusters is low, equal to 3) but it also indicates that, for example, predictions of records with high $\mathrm{NO}_{3}^{-}$are supported by only a specific portion of the overall training dataset.

Previous modelling has identified the most important factors influencing denitrification rate $(D a)$ to be: $T$, water filled pore space (WFPS), nitrate concentration $\left(\left[\mathrm{NO}_{3}^{-}\right]\right)$, and the soil denitrifying potential. The last factor can be either a long term (days, like LDP) or a short term (hours, like DEA) measurement. The short term denitrification potential metrics (DEA) are most commonly used. Although successfully used as a denitrification indicator (Heinen, 2006a), DEA techniques are varied and have an imprecise relationship to Da (Oehler et al., 2007; Simek et al., 2000).

In addition to the controlling factors outlined above (i.e. Temperature, WFPS, $\mathrm{NO}_{3}^{-}$and DEA), we tested the following factors:

- organic matter \% (OM): OM could be a useful surrogate for soil LDP which is correlated to soil physical characteristics more than DEA is (Simek et al., 2000). Some models use OM to compute a LDP (Hansen et al., 1991; Johnsson et al., 1987) which has been suggested to be more appropriate than DEA for modelling purposes (Henault and Germon, 2000).
Table 3. Contingency table of the counts of records per data source and cluster (k-medoid cluster), and the mean silhouette width per cluster (can be between $[-1,1]$, a score close to 1 meaning good clustering)

\begin{tabular}{rlll}
\hline k-medoid clusters & 1 & 2 & 3 \\
\hline Source & & & \\
Cosandey et al. (2003) & 3 & 52 & 0 \\
Henault and Germon (2000) & 18 & 19 & 2 \\
Luo et al. (1999) & 99 & 0 & 0 \\
Oehler et al. (2007) & 209 & 0 & 44 \\
Ryden (1983) & 46 & 0 & 0 \\
Zaman and Nguyen (2010) & 41 & 0 & 0 \\
\hline Medoids & & & \\
$D a$ & 0.05 & 0.05 & 0.54 \\
OM & 4.18 & 1 & 2.91 \\
SD & 0.1 & 0.1 & 0.3 \\
pH & 6.3 & 7.1 & 6.7 \\
WFPS & 63.6 & 87.3 & 53.7 \\
$T$ & 10.9 & 20 & 13.1 \\
NO & 27 & 32 & 322.91 \\
$D b$ & 1.1 & 1.5 & 1.57 \\
\hline Silhouette score & 0.33 & 0.20 & 0.40 \\
\hline & & &
\end{tabular}

- bulk density ( $D b)$ : Petersen et al. (2008) argued that gas diffusivity is affected by $D b$ and influences $\mathrm{O}_{2}$ concentration. This in turn strongly influences denitrification rates. Hence, it may be a better estimator of $\mathrm{O}_{2}$ concentration than WFPS. Moldrup et al. (2005) modelled gas diffusivity using soil porosity and pore size distribution which are correlated with $D b$, WFPS and OM.

- pH: soil pH is non-neutral toward denitrification with multiple direct and indirect effects (Simek and Cooper, 2002). Because of the use of the acetylene blockage technique for measuring denitrification, the influence of $\mathrm{pH}$ on nitrification rate and hence the supply of $\mathrm{NO}_{3}^{-}$ 
(Cheng et al., 2004; Hwang and Hanaki, 2000) is not taken into account.

- soil depth (SD): soil depth affects connectivity to the surface and hence influences aeration, $\mathrm{O}_{2}$ concentration and ultimately denitrification rate.

Three records from Cosandey et al. (2003) were discarded because they unbalanced the validation process, and strongly influenced the NEMIS model calibration.

\subsection{Boosted regression trees}

BRT are (after Elith et al., 2008) "an ensemble method for fitting statistical models that differs fundamentally from conventional techniques that aim to fit a single parsimonious model. Boosted regression trees combine the strengths of two algorithms: regression trees (models that relate a response to their predictors by recursive binary splits) and boosting (Schapire, 2003). The final BRT model can be understood as an additive regression model in which individual terms are simple trees, fitted in a forward, stagewise fashion". A k-fold cross-validation (CV) is used to avoid the effect of over fitting (over training) and assess the prediction performance. The training process is stochastic: it includes a random or probabilistic component (for example, sub-samples for $\mathrm{CV}$ are chosen randomly). This means that, unless a random seed is set initially, final models will be subtly different each time they are calibrated. BRT models can be fitted to a variety of response types (Gaussian, Poisson, binomial, etc.). The method is insensitive to outliers, and can accommodate missing data in predictor variables by using surrogates (Breiman et al., 1984). The final number of trees is controlled by two important factors: the learning rate (or shrinkage parameter) and the tree complexity.

One of the interesting features of BRT is the assessment of variable relative influences, based on the number of times a variable is selected for splitting, weighted by the squared improvement to the model as a result of each split, and averaged over all trees (Friedman and Meulman, 2003). The relative influence (or contribution) of each variable is scaled so that the sum adds to 100 , with higher numbers indicating stronger influence on the response. For a detailed example, see the working guide from Elith et al. (2008).

\subsection{Artificial neural networks}

The first mathematical representation of a neuron was introduced by McCulloch and Pitts (1943) with the perceptron. Each neuron receives input vectors $(\boldsymbol{X})$, performs a weighted sum $(\alpha)$, and through an activation (also called transfer) function $(G)$ (which may be linear or nonlinear) produces a result $(Y)$ in the form:

$Y=G(W X+b)$ where $W=\left(w_{i, 1}, w_{i, 2}, \ldots, w_{i, n}\right)$ are the neurons weights, $\boldsymbol{X}=$ $\left(x_{1}, x_{2}, \ldots, x_{N}\right)$ are the vector inputs of neuron $i, b$ is the neuron bias. $\alpha=(W \boldsymbol{X}+b)$ is the input weighted sum (also called potential of neuron $i$ ) and $G$ is the activation function. The classic non-linear activation function used is the sigmoid function:

$G(\alpha)=\left(1+e^{-\alpha}\right)^{-1}$

One or more neurons form a layer. In our study we used the common feed-forward ANN structure deriving from the perceptron, also called "multi-layer perceptron". The first neurons are forming the input layer, the lasts are forming the output layer, the others are forming one or more hidden layers (Hagan et al., 1996). The standard notation used throughout this work is [3:4:1], meaning 3 input nodes, 4 hidden and 1 output nodes (5 neurons). The number of input variables necessary for predicting the desired output variable determines the number of input nodes. The optimum number of hidden nodes and hidden layers is dependent on the complexity of the modelling problem. During training, patterns of input and corresponding output pairs are presented to the ANN, and the learning algorithm iteratively adjusts the values of connection weights within the ANN structure. It is desirable to attain the required level of accuracy with the simplest possible ANN structure (i.e., the fewest nodes) because this minimises training time, improves network generalization and lessens over-fitting effects (Hagan et al., 1996).

Potentially, different techniques could be used to "open" the ANN and try to understand the variable relationships (Gevrey et al., 2003). As suggested in the review from Gevrey et al. (2003), we chose the partial derivative method (Pad) (Dimopoulos et al., 1999) to assess the variable relative contribution to the output of the ANN.

\subsection{NEMIS model}

The NEMIS model uses a common formalism (Heinen, 2006b; Johnsson et al., 1987, 1991; Sogbedi et al., 2001):

$D a=D p \cdot f_{N} \cdot f_{S} \cdot f_{T}$

with

$f_{S}=\frac{N}{K+N}$

$f_{N}=\left(\frac{S-S_{t}}{S_{m}-S_{t}}\right)^{w}$

$f_{T}=Q_{10}^{\frac{T-T r}{10}}$

$D a$ is the denitrification rate $\left(\mathrm{mg} \mathrm{N} \mathrm{kg}^{-1}\right.$ soil d $\left.\mathrm{d}^{-1}\right)$ and $D p$ is the potential denitrification $\left(\mathrm{mg} \mathrm{N} \mathrm{kg}^{-1}\right.$ soil d$\left.{ }^{-1}\right)$. The denitrification potential can be either a LDP or a DEA. $f_{N}$ is a nitrate dimensionless function, where $N$ is the 
actual nitrate soil content $\left(\mathrm{mg} \mathrm{N} \mathrm{kg}^{-1}\right.$ soil) and $K$ is the nitrate soil content $\left(\mathrm{mg} \mathrm{N} \mathrm{kg}^{-1}\right.$ soil) when $f_{N}=0.5 \cdot f_{S}$ is a dimensionless function of water saturation, where $S$ is the WFPS, $S_{t}$ the WFPS threshold below which denitrification does not occur and $S_{m}$ the maximal WFPS (in our case $S_{m}=$ 1). $f_{T}$ is a dimensionless function of the soil temperature $T\left({ }^{\circ} \mathrm{C}\right), T r$ is the reference temperature when the potential denitrification $D p$ was determined, and $Q_{10}$ is the increase factor for a temperature increase of $10^{\circ} \mathrm{C}$. This function has a specific form in NEMIS, where two different $Q_{10}$ are used for two ranges of temperature:

$f_{T}=f T \operatorname{ref} \times Q_{10}^{\frac{T-T r}{10}}$

if $T \geq 10, Q_{10}=2, T r=20, f T$ ref $=1$ otherwise $Q_{10}=50$, $\operatorname{Tr}=10, f T$ ref $=0.5$. Temperatures are in ${ }^{\circ} \mathrm{C}$.

\subsection{Performance, confidence intervals and model developments}

To assess the prediction performance and confidence interval of both BRT and ANN, we used the bootstrap approach (Efron, 1987). Averages presented in the following sections could be seen as bagged predictors (Breiman, 1996), using the mean as a simple aggregator (i.e. the mean of the reponses of all the BRTS or ANNs corresponding to the boostrap replicates). These average (bagged) responses were only used here for presenting the reponse of the models and its accuracy. When presenting an non-averaged reponse of a model (e.g. for the performance graphs and the ANN model described in Appendix B), that model will be an individual BRT or ANN matching as close as possible the bagged response.

The BRT, ANN and NEMIS models were calibrated on the same subsets. The performance assessment and the validation of the model was done using the same approach:

- the modelling performance was evaluated using the conservative independent validation: the dataset was randomly subsampled into a calibration and a test subset.

- we used a resampling technique (bootstrap: the random subsampling and calibration is repeated many times) to estimate the distribution of the performance criterion and its mean. These estimated distributions have been used to compare different models/approaches.

The BRT training was done using the methodology and the R code from Elith et al. (2008). A number of different BRT models have been developed. We always retained three base variables: temperature, WFPS and $\mathrm{NO}_{3}^{-}$which previous studies have shown to be important. The nomenclature used is $\operatorname{BRTn}(X, Y)$ where $n$ is the number of input variables $(n \geq 3)$ and $X, Y$ are the independent variables included in addition to the 3 base variables. The suffix $G$ denotes that the model was trained on the whole (global) dataset. Thus
BRT5(OM,pH)G denotes a model using the 3 base input variables plus 2 others $(\mathrm{OM}$ and $\mathrm{pH})$ which was trained on the whole dataset. The BRT was specifically used to analyse the variable relationships. Different combination of input variables were tested, starting from a model using all the available variables, and then discarding the variables of lower importance until model performance significantly decreased.

The feed-forward ANN calibration was done using a classic method (using a training and a validation subset to control overfitting). To select the simplest ANN structure (with the fewest hidden nodes), we started with the number of nodes in the hidden layer equal to twice the number of input variables. We then decreased the number of nodes until there was a significant decrease in model performance (independent validation). The nomenclature used is the same as for BRT.

The NEMIS model (using DEA as the denitrification potential $D p$ ) was calibrated following a methodology adapted from Oehler et al. (2009) and Heinen (2006a). NEMIS was calibrated on the whole dataset (denoted NEMIS4G) without the Henault and Germon (2000) and Ryden (1983) datasets, because they contain no DEA measurements. NEMIS was also calibrated separately on each of the Zaman and Nguyen (2010), Oehler et al. (2007), Cosandey et al. (2003) and Luo et al. (1999) datasets (denoted NEMIS4Z, NEMIS4O, NEMIS4C and NEMIS4L).

More details about the calibration steps are available in Appendix A.

\subsection{Partial dependence and high dimensional plotting}

Graphical representation of the BRT or ANN response as a function of their arguments could provide a comprehensive summary of its dependence on the joint values of the input variables. Unfortunately, such visualization is limited to low dimensional arguments (reasonably up to three dimensions). For higher dimensions, an alternative is looking at a collection of plots, each one showing the partial dependence of the model response to different input variables.

As defined in Friedman (2001) and Friedman and Meulman (2003), the partial dependence function aims at representing (summarizing) the effect of a single variable across the entire variable space. Given the entire data space, a mean response to the considered input variable is computed. For example, to compute the partial dependence for OM, responses to $\mathrm{OM}$ variation are computed for the different combinations of $T$, WFPS, $\left[\mathrm{NO}_{3}^{-}\right]$and $\mathrm{pH}$ existing in the dataset, and are averaged. Hence, the partial dependence functions are built using all the data points. Although computationally intense, this can be also done for two input variables, leading to 3-D or contour plots. The magnitude of the partial dependence is somehow related to the relative influence: influent variables will tend to present high magnitudes. 


\subsection{Statistics}

The model performance criterion (or generalization error) used in this study was the Normalized Root Mean Square Error (NRMSE) defined as:

$\mathrm{RMSE}=\sqrt{\sum \frac{(s-o)^{2}}{n}}$

$\mathrm{NRMSE}=\frac{\mathrm{RMSE}}{\bar{o}}$

where $s$ are simulated values, $o$ the observed values, $\bar{o}$ the average of observed values and $n$ the size of the sample. We used the normalized criterion to enable comparisons between different sites and studies. For mean comparisons we used the parametric z-test. We also used the commonly used Pearson $r^{2}$. All the data processing, model developments and statistics were performed using the software " $R$ " version 2.10 (2008).

\section{Results}

\subsection{Relative influences of input variables as revealed by BRT and ANN}

The BRT was constructed using a number of trees varying from 1000 to 1500 , a learning rate of 0.01 and a tree complexity of 5. More complex structures were not found to increase prediction performance. The best ANN topology was always [ $N: 6: 1], N$ being the number of input factors $(N$ varied from 4 to 7) Using a large number of hidden nodes tended to give a better fit to the training dataset, but without gains for the test dataset (i.e. independent validation).

The BRT8(OM,pH,Db,DEA,SD)G mean model performance (NRMSE, independent validation) was 1.10. Figure 2 shows the relative influence of the different variables on the response. The variables are sorted from the most influencing: OM, WFPS, $T, \mathrm{NO}_{3}^{-}, \mathrm{pH}, D b$, DEA, SD. Scores for $T$ and WFPS were not significantly different (z-test, $p>0.05$ ). Simplification of the model down to 5 variables was done without significant loss of performance. Figure 3 shows the hierarchy of the variables for the BRT5(OM,pH)G model, which did not differ from BRT8(OM,pH,Db,DEA,SD)G. The relative influence of DEA did not change with or without Henault and Germon (2000) and Ryden (1983) records. Db, DEA and SD accounted for less than $10 \%$ of the influence, less than the influence of $\mathrm{pH}(12.2 \%)$. Reducing the BRT topology to 5 inputs did not shift the influence carried by the three discarded variables to a particular one. Discarding $\mathrm{pH}$ from the model did not increase the influence of $D b$, DEA and SD. Apparently, the effects of $D b$ and $\mathrm{pH}$ are independent, or at least treated as such in the BRT approach. Also, the

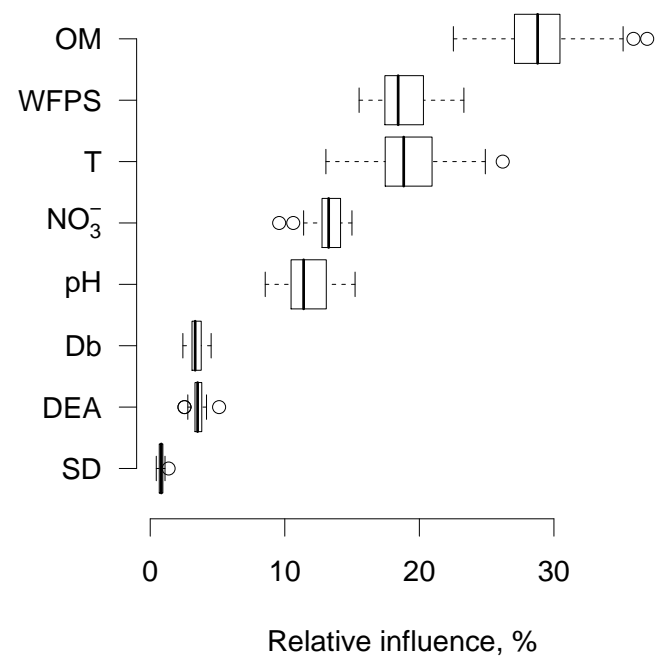

Fig. 2. Boxplots (description of quartiles with maximum at 1.5 interquartile range) of relative influences of the 8 tested input variables, as revealed by the BRT8(OM,pH,Db,DEA,SD)G model. Circles are outlier candidates.

importance of $\mathrm{NO}_{3}^{-}$is evident. As envisaged, the BRT approach successfully lessened dataset autocorrelation effects.

The ANN7(OM,pH,Db,SD)G mean model performance (NRMSE, independent validation) was 1.21. Figure 4 shows the relative influence of the different variables on the response. The variables are sorted from the most influencing: $\mathrm{NO}_{3}^{-}, T$, WFPS, OM, SD, $\mathrm{pH}, D b$. Scores for $D b, \mathrm{pH}$ and SD were not significantly different, as well as $T$ and OM $(p>0.05)$. Results of the ANN8(OM,pH,Db,DEA,SD)G (so without Henault and Germon (2000) and Ryden (1983)records) shown very variable results, with a slightly higher contribution of DEA compared to OM (average of $9 \%$ versus $7 \%, p>0.05$ ). Removing variables down to 5 changed the order of the variable contributions, with always a high value for $\mathrm{NO}_{3}^{-}$, and all the other being equal or less than $11 \%$. Simplification of the model down to five variables was done without significant loss of performance, whatever the fourth and fith variable was. ANN $4(\mathrm{OM}) \mathrm{G}$ and ANN4(DEA)G (without Henault and Germon (2000) and Ryden (1983)records) shown the same performance ( $p>$ 0.05 ). Figure 5 shows the hierarchy of the variables for the ANN5(OM,pH)G model. WFPS and OM influences were not significantly different $(p>0.05)$. As for the BRT approach, the importance of $\mathrm{NO}_{3}^{-}$is evident. The influence of the other variable is less clear. Overall the ANN results seemed to be quite sensitive to subsampling, and produced an unstable (highly variable) assessment of relative influences.

One of the main results is the possible replacement of DEA in favour of OM. Looking at the BRT analysis, OM appears to be a better candidate, more strongly than the small advantage for DEA in the ANN analysis. As already 


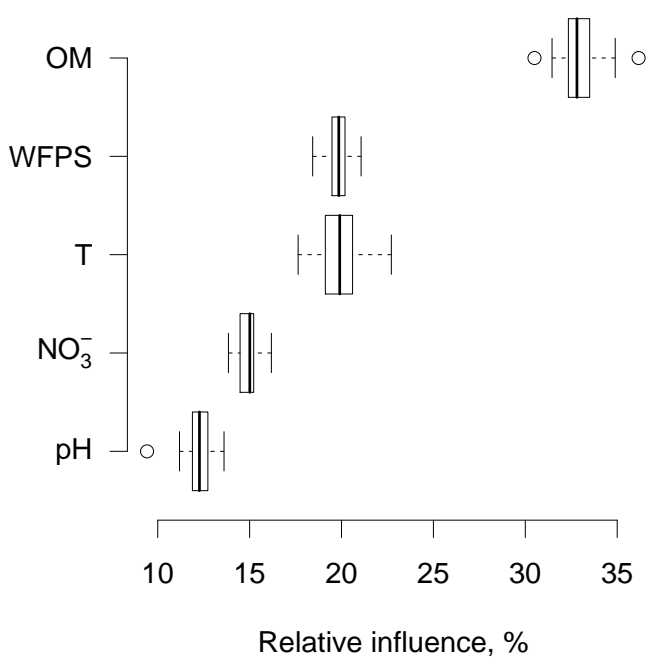

Fig. 3. Boxplot (description of quartiles with maximum at 1.5 interquartile range) of relative influences of the 5 input variables, as revealed by the BRT5(OM,pH)G model. Circles are outlier candidates.

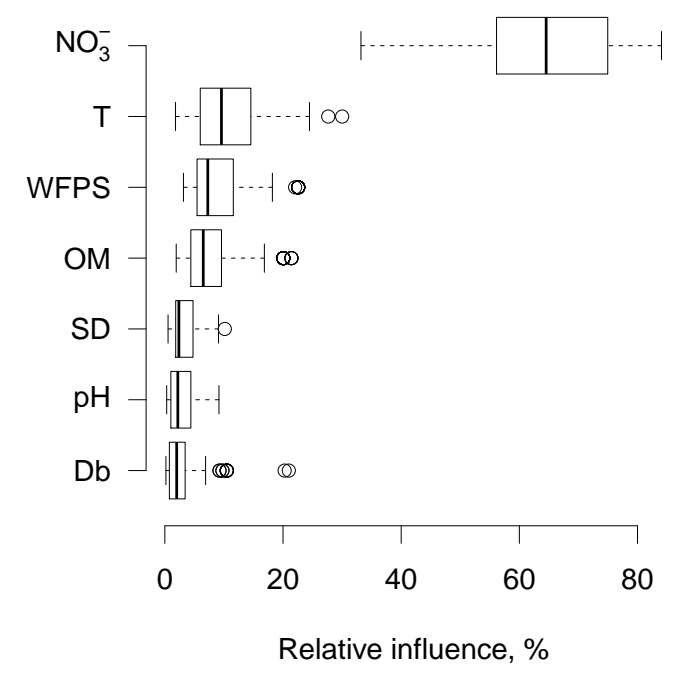

Fig. 4. Boxplots (description of quartiles with maximum at 1.5 interquartile range) of relative influences of the 7 tested input variables, as revealed by the ANN7(OM,pH,Db,SD)G model. Circles are outlier candidates.

stated, the three supplement variables $D b, \mathrm{pH}$ and $\mathrm{SD}$ are correlated (Table 2), and adding one of these to the model improves significantly the predictive performance of the model. Adding another one seems to add unnecessary complexity to the model, without performance gains. In our "final" model we decided to add $\mathrm{pH}$ to the base factors and OM. We rejected SD because the mechanism by which soil depth influences denitrification is unclear, and this variable has clearly a low influence. On the other hand, $\mathrm{pH}$ helped explaining variability more than $D b$.

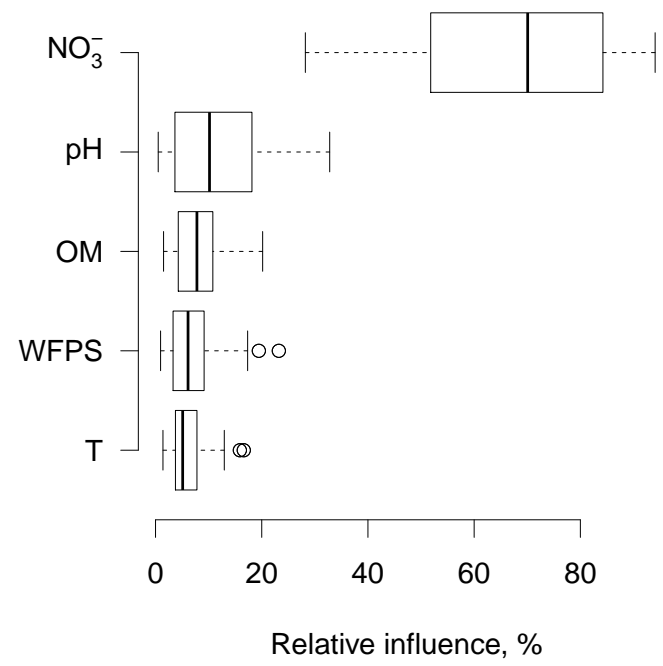

Fig. 5. Boxplot (description of quartiles with maximum at 1.5 interquartile range) of relative influences of the 5 input variables, as revealed by the ANN5(OM,pH)G model. Circles are outlier candidates.

\subsection{The BRT5(OM,pH)G, ANN5(OM,pH)G and NEMIS model performances}

Figure 6 shows the performances (independent validation with the test subset) of the BRT5(OM, pH)G model, the ANN5(OM, pH)G model and the NEMIS4G model. Removing Henault and Germon (2000) and Ryden (1983) records does not change the mean performance of the BRT and ANN models. The mean tests NRMSE are respectively $1.12,1.20$ and 2.07, and $r^{2}$ are $0.78,0.79$ and 0.28 clearly indicating that the BRT5(OM, pH)G and the ANN5(OM, $\mathrm{pH}) \mathrm{G}$ models result in better predictions (w-test, $p<0.05$, computed using Oehler et al. (2007), Cosandey et al. (2003), Luo et al. (1999) and Zaman and Nguyen (2010) but without Henault and Germon (2000) and Ryden (1983) records because they do not include DEA which is a required input in NEMIS). Heinen (2006a) already pointed out that NEMISlike models can perform quite well when calibrated for a specific site, but that they do not perform well when applied over a range of different soil types with the same parameter set. The site-specific calibrated NEMIS on the Oehler et al. (2007), Cosandey et al. (2003), Luo et al. (1999) and Zaman and Nguyen (2010) datasets (NEMIS4O, NEMIS4C, NEMIS4L, and NEMIS4Z) showed that model coefficients (notably those relating denitrification rate to WFPS) varied significantly among datasets. Consequently, when NEMIS was calibrated using all 4 datasets (NEMIS4G) it did not perform particularly well. There is only a slight difference in prediction performance (z-test, $p<0.05$ ) between the BRT5(OM, pH)G and the ANN5(OM, pH)G models. Looking at the range of the Test NRMSE (roughly between 0.5 and 1.7 for BRT, 0.8 and 2.6 for ANN, and 0.7 and 3.5 for NEMIS), there seems to be a rather high influence 


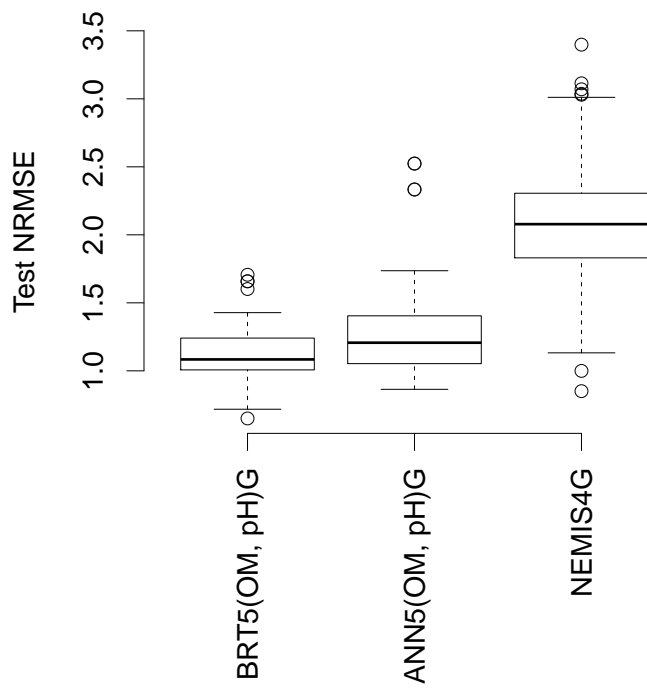

Fig. 6. Boxplot (description of quartiles with maximum at 1.5 interquartile range) of prediction performance (independent validation) of ANN5(OM, pH)G, BRT5(OM, pH)G and NEMIS4G (without Henault and Germon (2000) and Ryden (1983) records). Circles are outlier candidates.

of the sub-sampling process on the independent validation. This can be due to the lack of data (67 records for the Test dataset) coupled to the presence of few extreme $D a$ values that can have a relatively large impact on the independent validation process. In contrast, the Training NRMSE min and max values are relatively low for both BRT and ANN (between 0.70 and 1.00 for a mean of around 0.80 ). The BRT shows more stable results, maybe thanks to the ensemble technique.

Figure 7 presents the performance of the chosen BRT5(OM, pH)G, ANN5(OM, pH)G and NEMIS4G. ANN NRMSE for each dataset from Oehler et al. (2007), Henault and Germon (2000), Luo et al. (1999), Cosandey et al. (2003), Ryden (1983) and Zaman and Nguyen (2010) are respectively $1.36,0.63,0.73,0.46,1.21$ and 0.85. ANN5(OM, pH)G and BRT5(OM, pH)G display a comparable behaviour. Figure 7 highlights the meaning of the differences in performance (from NRMSE of 2.07 to 1.20) between the NEMIS4G model and the ANN5(OM, $\mathrm{pH}) \mathrm{G}$ model. The ANN model clearly outperforms the NEMIS model. Site specific calibration of NEMIS gives average NRMSE (computed on the whole dataset, not independent ones) for Oehler et al. (2007), Luo et al. (1999) Cosandey et al. (2003) and Zaman and Nguyen (2010) of $1.55,0.66,1.03$ and 1.07 , to be compared with 1.36, $0.73,0.46$ and 0.87 for ANN5(OM, Db)G. Overall, the ANN5(OM, pH)G model seems to be at least as good as (z-test, $p>0.05$ for Luo et al., 1999), if not better (w-test, $p<0.05$ for Oehler et al., 2007, Cosandey et al., 2003) and Zaman and Nguyen (2010), than the site specific NEMIS models.

\subsection{The BRT5(OM,pH)G and ANN5(OM,pH)G model response shapes}

In Figs. 8 and 9 we show the univariate and bivariate partial dependence plots for the BRT5(OM,pH)G and ANN5(OM,pH)G models.

The 95\% confidence intervals are displayed in Fig. 8. These reflect both the stability of the results and somehow the density of the datapoints. Dataset boundaries are shown in Fig. 8 using rug ticks and in Fig. 9 using convex hulls. As guidelines to evaluate data point distribution, scatter plots of the combinations of $\mathrm{Da}, \mathrm{NO}_{3}^{-}$, WFPS, OM, $\mathrm{pH}$ and $T$ are available in the Appendix D.

Overall, BRT and ANN shown the same trends (Fig. 8). Although not smooth (due to the piecewise constant approximation), BRT gave more stable results than ANN (tighter confidence intervals). This may be thanks to the ensemble approach of the BRT.

\subsubsection{Influences of $T$ and WFPS}

The response shapes of WFPS and $T$ (Fig. 8a and 8c) are similar to the description proposed by NEMIS types of models. In details, the BRT predicts a small peak around $10^{\circ} \mathrm{C}$. Whatever the values of other variables, there are clear threshold values: below $10^{\circ} \mathrm{C}$ and a WFPS of $50 \%$, predicted $D a$ rate is very low, and nearly null for WFPS $<30 \%$. On average, at around a WFPS of $80 \%, 50 \%$ of the maximal $D a$ rate is achieved. The ANN response to $T$ below $5{ }^{\circ} \mathrm{C}$ is quite unstable (high confidence intervals). This may show some of the limits of the datasets which included no records with very low or null $T$. WFPS and $T$ are always limiting factors (Fig. 9).

\subsubsection{Effects of the substrates $\mathrm{NO}_{3}^{-}$and $\mathrm{OM}$}

$\mathrm{Da}$ response to $\left[\mathrm{NO}_{3}^{-}\right]$variation (Fig. 8b) is (also) similar to NEMIS. BRT and ANN shapes are similar, but the ANN predicts a higher response, on average. The ANN partial dependence is also quite unstable (high confidence intervals). Very low $\mathrm{NO}_{3}^{-}$concentrations still induce a relatively high $D a$. Also, above a $\left[\mathrm{NO}_{3}^{-}\right]$of 200 , and up to $800 \mathrm{mg} \mathrm{N} \mathrm{kg}^{-1}$ soil, the response is not a straight plateau line, but quickly decreases. Again, this shows some of the limits of the dataset which included no records with very low or null $\left[\mathrm{NO}_{3}^{-}\right]$, and data are very scarce for $\left[\mathrm{NO}_{3}^{-}\right]>200 \mathrm{mg} \mathrm{N} \mathrm{kg}^{-1}$ soil (Fig. 1, top middle panel) especially around $20^{\circ} \mathrm{C}$.

The response to OM is nearly linear for ANN with $D a$ rate increasing with OM \% (Fig. 8d). The BRT presents a stepper result, with a jump around $7.4 \%$. Looking at Fig. 9c, $\mathrm{NO}_{3}^{-}$ and $\mathrm{OM}$ effects seem rather independent and additive. The behaviour of the model near values of 0 may seem odd. As we are modelling a non-dynamic $D a$ rate, beside an artefact effect due to a lack of data (particularly true for low OM), 

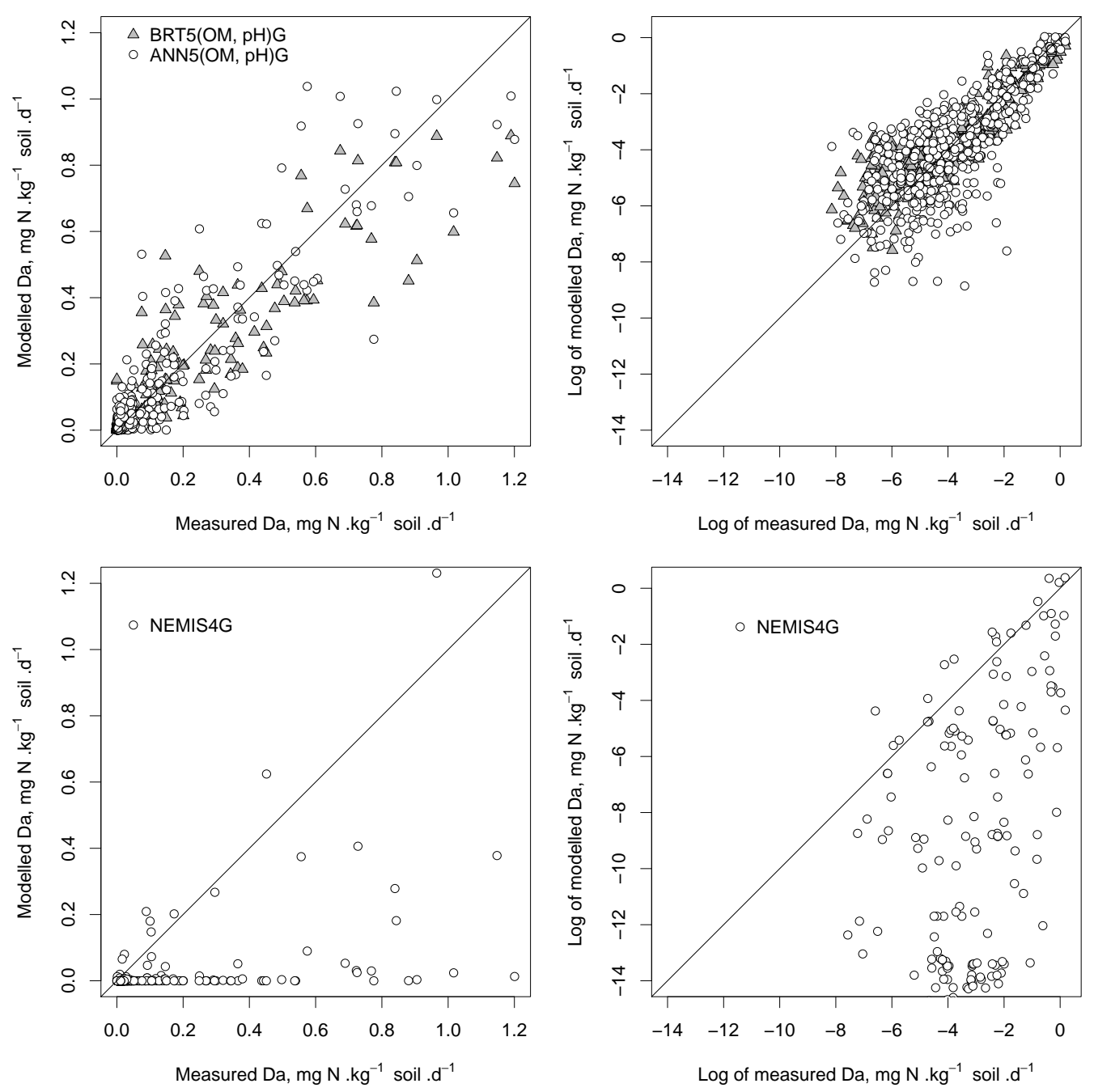

Fig. 7. Comparison of BRT5(OM, pH)G, ANN5(OM, pH)G and NEMIS4G (whole dataset without Henault and Germon (2000) and Ryden (1983) records) $D a$ prediction performance (independent validation).

the model simply predicts that denitrification starts at very low levels of $\left[\mathrm{NO}_{3}^{-}\right]$and OM. Also some low $\mathrm{NO}_{3}^{-}$supply from nitrification process might have also occured, even if measurements were performed using the $\mathrm{C}_{2} \mathrm{H}_{2}$ blockage technique which in principle should inhibit nitrification. In practice, when included in a dynamic model the overall denitrified $\mathrm{N}$ will be very low in such conditions, with $\mathrm{NO}_{3}^{-}$ being quickly depleted.

\subsubsection{Influence of $\mathrm{pH}$}

The partial dependence shapes and location (i.e. the mean response) for $\mathrm{pH}$ are quite different between BRT and ANN. Though, they display the same trends, and both show a maximum for a $\mathrm{pH}$ of around 7.2 (Fig. 8e). The decrease after this maximum value toward alkaline condition is supported by few records, and the BRT, and especially the ANN results are very unstable in this area. For the same reasons, no strong conclusions can be drawn for $\mathrm{ph}<6$ (e.g. an horizontal line from $\mathrm{pH} 6$ to the smallest value can be drawn inside the $95 \%$ confidence intervals). Figure $9 \mathrm{~d}$ and $9 \mathrm{j}$ shows the partial dependence of $\mathrm{pH}$ with $\mathrm{NO}_{3}^{-}$and $\mathrm{OM}$, with again a maximum around a $\mathrm{pH}$ of 7.1. This value is coherent with what has been found in the literature (see Simek and Cooper, 2002). However, looking at the details in Fig. 9d, $9 \mathrm{~g}, 9 \mathrm{i}$ and $9 \mathrm{j}$, this maximum can depend on the conditions, in particular $\mathrm{pH}$ impact seems to be a function of $T$ (or rather the other way round, as it is unlikely to see fast variations of $\mathrm{pH}$ in soils), with maximum values going down from $T / \mathrm{pH}$ of $20^{\circ} \mathrm{C} / 7.2$ to $10^{\circ} \mathrm{C} / 6.6$. These results are also present in the BRT5(OM,pH)G model. As pH represents different types of soils, this might be the expression of different microorganism populations, or as suggest by Simek and Cooper (2002), the $\mathrm{pH}$ influences many facets of the denitrification process. 

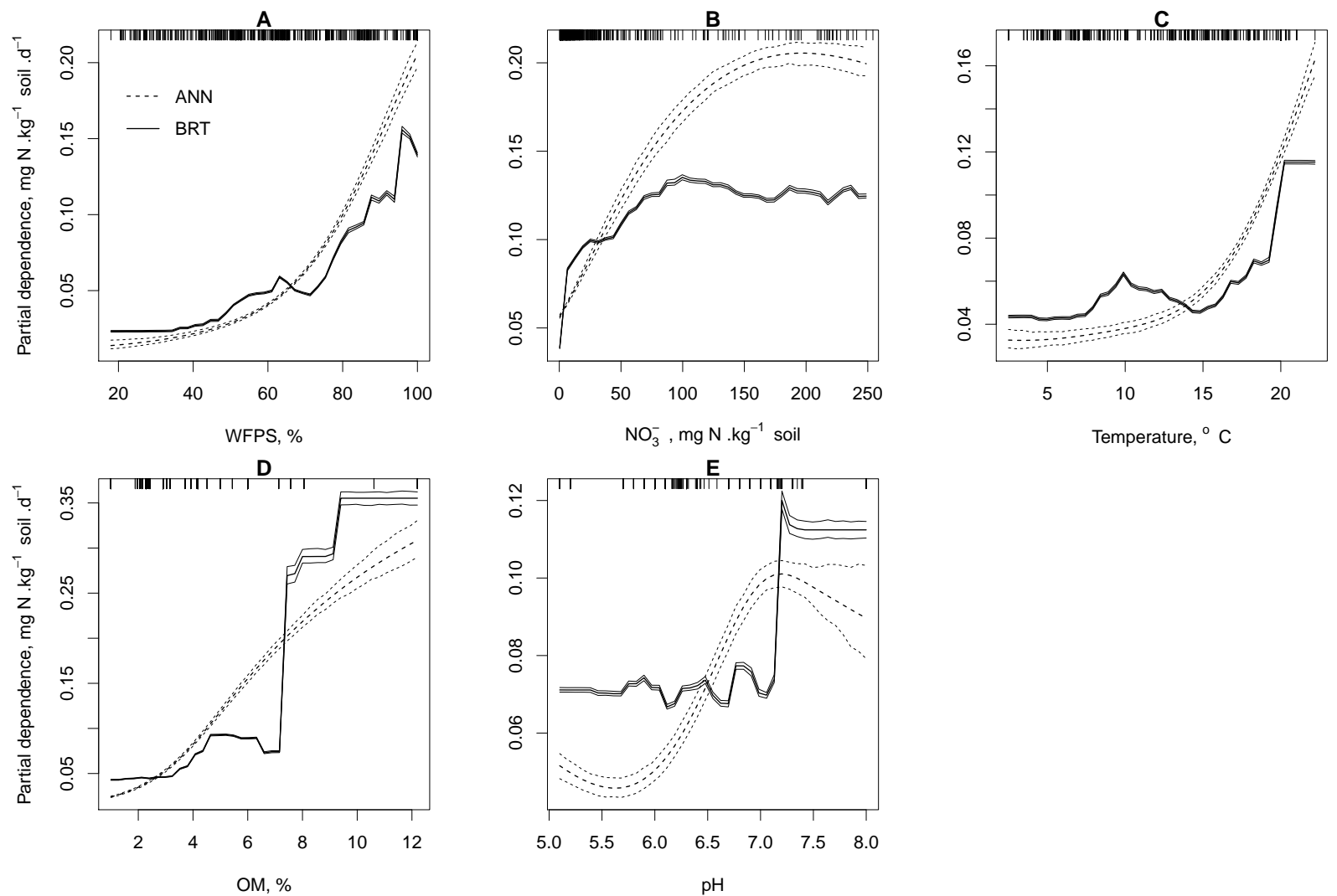

Fig. 8. Mean partial dependence of BRT5(OM,pH)G and ANN5(OM,pH)G models, with 95\% confidence intervals (bootstrap). Rug plots on each subplot indicate the presence of the $\mathrm{x}$-axis data in the dataset (e.g. only few different values of $\mathrm{pH}$ are present in the original dataset and so few lines appear in the rug plot of subplot E).

\section{Discussion}

Our work was first motivated by concerns on the predictive performances of widely used simplified model such as NEMIS. The use of ML methods not only provided a more performant denitrification model, but may have also shed new light on denitrification processes, especially with respect to the relative influence of factors and how they interact.

The BRT method has very good predictive performance, results are relatively stable, easier to interpret and the training is faster. But we can see some issues for efficient uses as a predictive model: the model response is not smooth, and because of portability and mainly computing time issues, it cannot be easily and efficiently implemented in field or larger scale models. The ANN calibration method aimed at reducing the effect of initial conditions by repeating training and sub-sampling, and by carefully assessing the prediction performance using bootstrapping. This is relatively timeconsuming but is essential.

Analysing the variable relative influence using ANN with the $\mathrm{PaD}$ approach gave quite variable results with only one clear influence $\left(\mathrm{NO}_{3}^{-}\right)$. With this technique, using the ANN alone would have been of little help on this "noisy" dataset (high denitrification measurement errors). This may be explained by one of the weakness of ANNs: they are particularly unstable predictors. Small changes in the training data set may produce very different models (Breiman, 1996; Cunningham et al., 2000) and consequently different performance on unseen data. Breiman (1996) suggests that these different models may result from the training of the ANN getting caught in different local minima in the error surface. This lack of stability is unknown a priori (it depends on the training dataset) and tends to limit the generalisation capability or performance of ML (Bousquet and Elisseeff, 2002). Overall, the usefulness of ANN to analyse variable influences is limited in certain cases (Olden et al., 2004).

When applied to Regression Trees or ANNs, ensemble techniques can produce significant improvements in generalization capability and, consequently, of the overall performance (e.g. Pasti et al., 2010; Perrone and Cooper, 1993; Friedman, 2001). There are many different ensemble techniques, the most popular include bagging (Breiman, 1996) and/or boosting (Schapire, 2003; Freund and Schapire, 1995). Bagging (for "bootstrap aggregation") uses the bootstrap statistical re-sampling technique (Efron, 1987), 

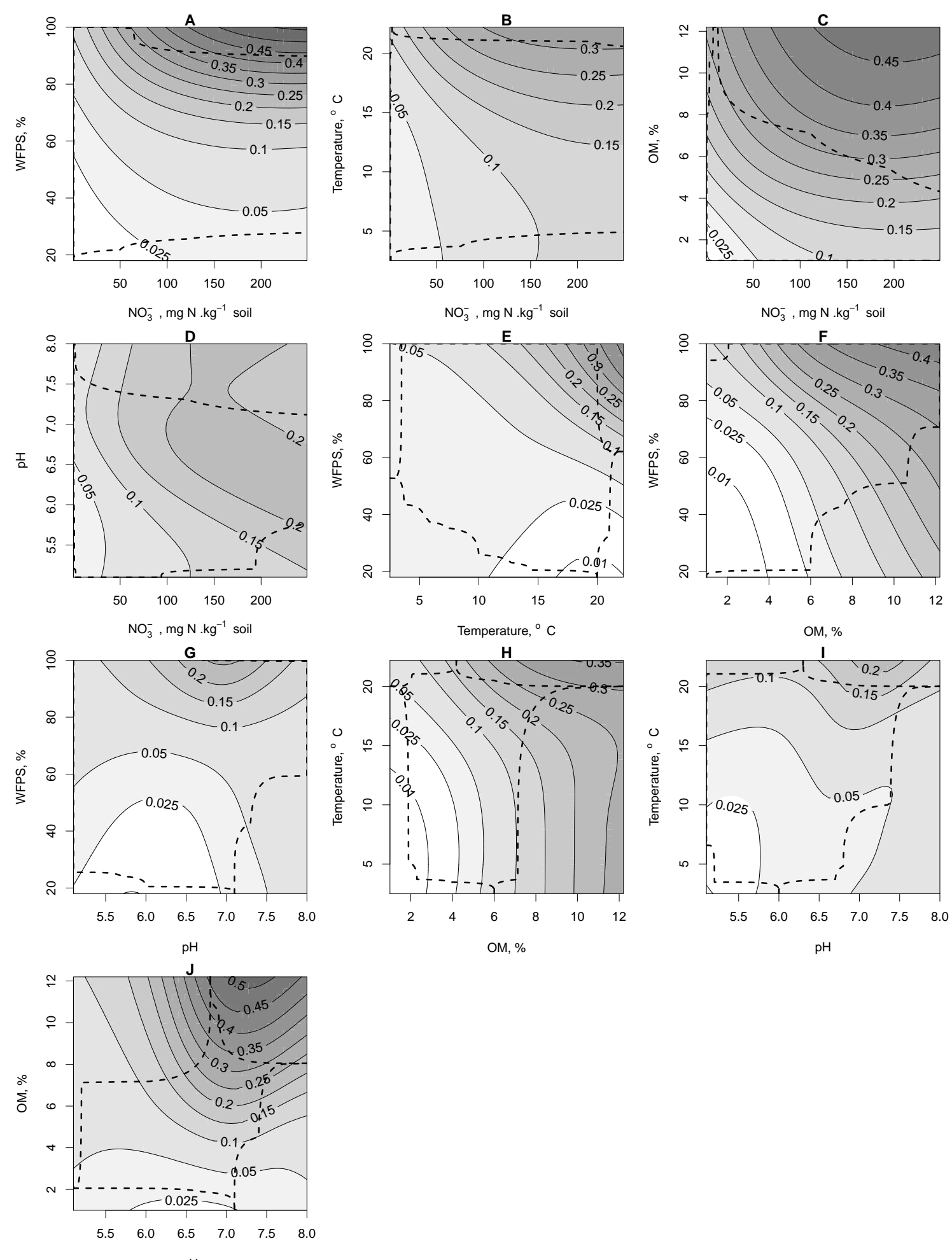

$\mathrm{pH}$

Fig. 9. Mean bivariate partial dependence of ANN5(OM,pH)G models, in $\mathrm{mg} \mathrm{N}^{-1}$ soil d ${ }^{-1}$. Dashed lines delineate the presence of data points, using a generalisation of the convex hull (Gagolewski, 2009). 
to generate multiple training sets and ML sub-models (e.g. a single regression tree or ANN) for an ensemble. Bagging has a number of key advantages, one of the most important is the ease with which confidence intervals can be computed (Carney and Cunningham, 1999; Tibshirani, 1996). Boosting is based on an adaptive method to combine many simple "weak" models to give improved predictive performance (e.g. AdaBoost, Freund and Schapire, 1995). BRT is an ensemble version of single regression trees, constructed using the boosting technique. As such they often provide a more stable prediction and a more accurate performance assement than single ANNs. That could explain the clearer outlook of the results obtain with BRT.

The two ML models perform better than NEMIS on our extended database (which includes data from uplands and wetlands in intensive and less intensive agrosystems but with relatively uniform (loamy) soil types). This is also true for BRT4(DEA)G and ANN4(DEA)G models (NRMSE of 1.31 and 1.36, z-test, $p<0.05$ ) which use the same inputs as NEMIS, or even for BRT3G and ANN3G models (using only the base variables $\left[\mathrm{NO}_{3}^{-}\right]$, WFPS and $T$, NRMSE of 1.52 and 1.63 , z-test, $p<0.05$ ), this later models exhibiting overall the same trends. To be fair with the NEMIS model, it is to be noted that NEMIS was originally designed to use a LDP, which is obviously quite a different method to evaluate denitrification potential than DEA. However, the model has been successfully used with DEA measurements (see Heinen references for more details).To check that the conjugate gradient method used for NEMIS optimisation was not underperforming, we also tried other techniques such as differential evolution, but results did not improve significantly.

Overall, though presenting a $r^{2}$ of around 0.78 , the generalization errors (or performances) are still around $110 \%$. The performance seems to be mainly impaired by the Oehler et al. (2007) dataset. The main characteristics differentiating this dataset from the others are the presence of different soils and that measurements have been obtained in natural conditions (low temperatures), exhibiting the lowest $D a$. There might be a real effect not captured by the ML algorithms or not contained in the tested input variables. It is also possible that the relatively high measurement errors associated with low gas concentrations could be the cause of discrepancies in the prediction performance or reflect a limitation of the $\mathrm{C}_{2} \mathrm{H}_{2}$ blockage technique, especially on these low drainage soils.

Using a classic independent validation and the NRMSE score, the generalization error of the model was only assessed inside the training dataset space. The model should not be used outside its validity range (so not for extrapolation), and the results are limited to this range. Of course this is true for any model, but particularly for ML models which are strongly nonlinear and often display unrealistic behaviours outside their domain of validity. Intuitively, the level of generalization of the model is related to the data density in each part of the data space. Denser data points are needed to represent fast gradient change area. Without external knowledge, we cannot know if the gradients are well represented: indeed, they are built with the data (training). If the proposed ANN5(OM, pH)G was to be used, the limits of its applicability (i.e. the limits of the database) are provided on Fig. 1, Fig. 8, Fig. 9 and in the Appendix Fig. D1. The extreme limits (minimum and maximum of each input variables) are coded into the ANN formula (i.e. the scaled input values of the input vector should not be outside [0-1]).

The paucity of denitrification measurements $\left(\mathrm{N}_{2}+\mathrm{N}_{2} \mathrm{O}\right)$ in terrestrial environemnts, and especially in riparian zones, has been often highlighted (e.g. see Seitzinger et al., 2006; Hofstra and Bouwman, 2005; Groffman et al., 1998, 2006; Haag and Kaupenjohann, 2001; Machefert et al., 2002; Fisher and Acreman, 2004; Basset-Mens et al., 2006). Our database was built with measurements using the $\mathrm{C}_{2} \mathrm{H}_{2}$ blockage technique, mainly because of the availability of the data comprising the full set of variables we wanted to test (e.g. with WFPS, DEA, pH...), in wetland, riparian and upland terrestrial area. We chose to not mix measures obtained with different techniques (i.e. the isotope ones). Consequently this choice excluded notably soils with very low $\left[\mathrm{NO}_{3}^{-}\right]$, like forest's, where denitrification can not be correctly assessed with the $\mathrm{C}_{2} \mathrm{H}_{2}$ technique (Groffman et al., 2006). Nonetheless, the method we propose can be used with datasets using the isotope techniques. These measurement methods are becoming more and more affordable and widespread (Groffman et al., 2006), and hopefully we will be able to build better models using these data which have the potential to span broader environmental conditions with reduced measurement errors (Bollmann and Conrad, 1997; Groffman et al., 2006).

At first glance, the BRT and ANN results agree with the mathematical representation of NEMIS like models, which were already capturing the main effects (beside $\mathrm{pH}$ ). In details, contrary to ML models, NEMIS does not take into account non-linear variable interactions, such as temperature impact on each factor, which are more subtle than a linear effect. This is needed to efficiently simulate denitrification in real world conditions, where input variables are not at the higher end of their range (e.g. $20^{\circ} \mathrm{C}, 100 \%$ WFPS, $200 \mathrm{mg} \mathrm{N} \mathrm{kg}^{-1}$ soil, $10 \% \mathrm{OM}, \mathrm{pH} 7$ ) as often explored in laboratory-controlled experiments (e.g. the ones used to build NEMIS). Measurements tend to be less precise as we measure low $D a$ rates, and measurement biases and errors tend to be more impacting (e.g. limit of the sensor sensitivity, leaks or contaminations becoming more important, impact of nitrification inhibition if low $\left.\left[\mathrm{NO}_{3}^{-}\right]\right)$. ML are less sensitive to data noise, and this might also explain why they perform better on the low $D a$ range.

Overall, we think the main significance of this contribution is methodological: with ML approaches (or other modelling approaches like the generalized linear models or the additive linear models) different experimental design (other 
than controlled laboratory experiments) could be used to understand processes, especially at larger scales (e.g. catchment). The better representation of small $D a$ rates may also have an impact on our understanding of the $N$ cycle dynamic at the catchment scale, mainly because the unsaturated areas can represent the vast majority of the total surface. As the problem is non-linear and spatial interactions are crucial, this would have to be thoroughly tested combining ML with spatially distributed models.

ML approaches are interesting tools to study single variable effects, and, if enough data is available, they may not need measurements from experiment specifically designed to study the impact of separate factors. They are particularly useful to analyze and design models with data from surveys based on stratified experiment approaches (i.e. gradients sampling). As such, when using ML as an analysis tool the main objective when collecting data is to capture gradients, the most possible variability in all the variable spaces. To develop a NEMIS like model, a classic laboratory controlled experiment where all the variables are fixed but one was used. Generally, more measurements are needed for a ML analysis. However data can be obtained from surveys and not only from manipulative experiments, and may be more representative of the studied process in his "non-disturbed" environment. Moreover, interactions are more likely to be captured. After a ML based analysis, if the process and variable relationships are better understood, a simpler mathematical representation can be formuled.

The BRT analysis reaffirms the importance of temperature, WFPS and $\mathrm{NO}_{3}^{-}$, and highlights the importance of $\mathrm{OM}$ and pH. Our results and other works (Cosandey et al., 2003; Simek et al., 2000, 2002) indicate that the relationship between DEA and $D a$ is unclear. We successfully used OM instead of DEA without performance loss. This is consistent with the findings of Cosandey et al. (2003), who suggested that the proximal factors, available $\mathrm{OM}, \mathrm{O}_{2}$ and $\mathrm{NO}_{3}^{-}$, exert a stronger control on denitrification rates than the size of the denitrifying enzyme pool. As we used the Cosandey et al. (2003) dataset, we checked separately its impact on BRT results. It appears that without the Cosandey et al. (2003) data, OM and DEA have the same relative influences. The Cosandey et al. (2003) dataset shows the widest $\mathrm{OM}$ range and the highest $\mathrm{OM}$ values in our database. As the sampling of the gradients is not uniform across the datasets, this particular dataset might have biased the results while representing only $11 \%$ of the records. Also DEA measurements may be less precise than OM measurements. This might have led the BRT analysis to favour OM, even if it is relatively resistant to data noise. However Cosandey et al. (2003) dataset presents a larger range of values and there is no clear trend in favour of DEA without this dataset. More recently, apart from the Cosandey et al. (2003) conclusions, Miller et al. (2008) suggested that $D a$ is decoupled from the denitrifier community abundance. Overall, DEA does not seem to be a better indicator of $D a$ rate than OM, especially in agrosystems where supply of $\mathrm{NO}_{3}^{-}$is frequent and denitrifier communities are already adapted to their environment. An interesting implication is the integration of a feedback loop from soil organic carbon long term dynamic.

Another relevant result is related to the effect of $\mathrm{pH}$. This factor may be the one which has to be taken into account to differentiate soils. The cause-effect relationship between $\mathrm{pH}$ and denitrification remains unclear, even though a through review (Simek and Cooper, 2002) has clearly shown that such an effect is indeed present and should be accounted for. $\mathrm{pH}$ might also be important when estimating $\mathrm{N}_{2} \mathrm{O}$ emissions because $\mathrm{pH}$ affects the $\mathrm{N}_{2} / \mathrm{N}_{2} \mathrm{O}$ ratio (Firestone et al., 1980). This would have to be confirmed with a larger dataset, as the separation of $\mathrm{pH}$ and $\mathrm{Db}$ may not be sufficient. We will need to widen the database to other more contrasted type of soils (with more clay notably) and more records to fill gaps in the gradients and lessen dataset effects. This will improve prediction accuracy and increase model generalization.

BRT and ANN might be promising approaches for $\mathrm{N}_{2} \mathrm{O} /\left(\mathrm{N}_{2}+\mathrm{N}_{2} \mathrm{O}\right.$ ) modelling as well (soil $\mathrm{N}_{2} \mathrm{O}$ emission modelling with ANN has already been successfully performed, but not specifically from denitrification; Ryan et al., 2004). The next obvious step will be coupling the ANN model to a catchment scale model.

\section{Appendix A}

\section{Detailed calibration routines}

\section{A1 BRT calibration}

The BRT training was done using the R code from Elith et al. (2008), which uses the package gbm (Ridgeway, 2007). The methodology used is outlined in the following steps:

1. scaling of input and output variables using the same procedure as for the ANN calibration.

2. randomly sub-sampling the dataset to give 2 subsets: Training and Testing (in the proportion 7/8 and 1/8 of samples). The Training subset is used for the training (calibration) phase, and the Testing subset is used for independent validation.

3. training/validation of a BRT using a Gaussian response.

4. steps 2 to 3 were repeated 800 times. This resampling method enabled us to estimate the distribution of the performance criterion, provided confidence intervals for the calibration and prediction process and allowed for statistical model comparisons (Bootstrapping).

5. as a representative BRT, we selected the one closest to the mean (bagged) model (distance evaluated using the RMSE). 
The outputs are used after being transformed back and scaled back to the original data space (the performance is evaluated in the original data space).

\section{A2 ANN calibration}

The ANN training was done using the package AMORE (Pernía-Espinoza et al., 2005). The methodology used is outlined in the following steps:

1. scaling the input variable:

$$
x_{i, \text { scaled }}=\frac{x_{i}-\min _{x}}{\max _{x}-\min _{x}}
$$

Scaling is not mandatory for the input variables, but can ease further analysis of the trained ANN weights and biases.

2. scaling the output variable: a simple linear scaling (between 0 and 1), a log transformation and an arcsine transformation of the response variable were tested and all resulted in similar prediction performance. The arcsine transformation was finally chosen because it exhibited a more normal distribution of the residuals and was not over fitted on the highest values. Specifically, the arcsine transformation implies:

$$
\begin{array}{r}
x_{i, \text { transformed }}=\operatorname{arcsine}\left(\sqrt{\frac{x_{i}-\min _{x}}{\max _{x}-\min _{x}}}\right) \\
\times \frac{360}{2 \pi \times 100}
\end{array}
$$

The principal characteristic of the arcsine transformation is to stretch the low and high values, and condense the medium range values. The scaling between 0 and 1 for the response variable is mandatory for ANN, as the output of the ANN is between these values (sigmoid function).

3. randomly sub-sampling the dataset to give 3 subsets: Training, Validation, Testing (in the proportion 6/8, 1/8, $1 / 8$ of samples). The Training and Validation subsets are used for the training (calibration) phase, and the Testing subset is used for independent validation.

4. training/Validation of a feed-forward ANN. The learning algorithm used was the adaptive gradient descend with momentum, using the robust Least Mean Log Square criterion (Liano, 1996). The ANN was initialized with random weights and bias. Over-training was controlled by the validation subset.

5. step 4 was repeated 22 times with different initial conditions of weights and biases. This is necessary because the initial conditions of the ANN weights and biases are not neutral and can affect the prediction accuracy of the algorithm. Specifically, assuming an a priori normal distribution, we have used the following approach: (a) we want to be in the $10 \%$ best cases $(P)$ (b) we want to be in that case with a confidence of $90 \%$ ( $P$ conf) That gives $\mathrm{n}$ such as:

$(1-P)^{n} \leq P \operatorname{conf} \Longrightarrow n \geq 22$

Only the best combination of validation and training NRMSE was retained.

6. steps 3 to 5 were repeated 800 times. The number of times a step was repeated results from a trade off between statistical significance and computing time. This resampling method enabled us to estimate the distribution of the performance criterion, provided confidence intervals for the calibration and prediction process and allowed for statistical model comparisons (Bootstrapping).

7. as a representative ANN, we selected the one closest to the mean (bagged) model (distance evaluated using the RMSE).

The outputs are used after being transformed back and scaled back to the original data space (the performance is evaluated in the original data space).

\section{A3 NEMIS calibration}

The NEMIS model (using DEA as the denitrification potential $D p$ ) was calibrated following a methodology adapted from Oehler et al. (2009) and Heinen (2006a):

1. randomly sub-sampling the dataset to give 2 subsets: Calibration and Testing (in the proportion 7/8 and 1/8 of samples). The Calibration subset is used for the calibration phase, and the Testing subset is used for independent validation.

2. calibration of a NEMIS model (minimising the RMSE with a gradient descent algorithm).

3. steps 1 to 2 were repeated 800 times. This resampling method enabled us to estimate the distribution of the performance criterion, provided confidence intervals for the calibration and prediction process and allowed for statistical model comparisons (Bootstrapping).

4. as a representative NEMIS model, we selected the one closest to the mean (bagged) model (distance evaluated using the RMSE). 


\section{Appendix B}

\section{ANN model equation}

The sygmoid transfer function:

$G(\alpha)=\left(1+e^{-\alpha}\right)^{-1}$

The full ANN equation:

$$
D a=\sin \left(\frac{G\left(W_{o} \times G\left(W_{h} \times X+b_{h}\right)+b_{o}\right)}{\frac{360}{2 \pi \times 100}}\right)^{2}
$$

with the input vector

$$
\boldsymbol{X}=\left[\begin{array}{c}
\frac{\mathrm{NO}_{3}^{-}-0.34}{759.66} \\
\frac{\mathrm{WFPS}-17.985}{82.015} \\
\frac{\text { Temperature-2.5 }}{19.7} \\
\frac{\mathrm{OM}-1}{11.2} \\
\frac{\mathrm{pH}-5.1}{2.9}
\end{array}\right]
$$

the weight matrix of the hidden layer

$W_{h}=\left[\begin{array}{rrrrr}-12.5941098 & 1.3397349 & -0.3600218 & -1.6165129 & 1.3481651 \\ 5.743649 & -4.432043 & 1.861159 & -7.962878 & 5.469261 \\ 5.0120164 & -1.2927237 & 0.1530118 & -1.1013582 & -0.262014 \\ -2.301329 & -3.834269 & -7.255529 & 2.646514 & 6.241783 \\ 3.334881 & -1.879438 & 10.067627 & -1.289163 & -11.724987 \\ -5.612255 & 4.181079 & -3.514725 & -1.067258 & -6.007714\end{array}\right]$

and its bias

$$
b_{h}=\left[\begin{array}{r}
-2.512686 \\
0.1410123 \\
-0.5807459 \\
3.895448 \\
-0.01380899 \\
2.363688
\end{array}\right]
$$

the weight matrix of the output layer

$W_{o}=[-7.2950421-2.5771119-0.1618821-4.295324-3.7707312-2.505269]$

and its bias

$b_{o}=[4.826489]$

In the above, $D a$ is denitrification rate $\left(\mathrm{mg} \mathrm{N} \mathrm{kg}^{-1}\right.$ soil d$\left.^{-1}\right)$, $\mathrm{NO}_{3}^{-}$is nitrate soil concentration $\left(\mathrm{mg} \mathrm{N} \mathrm{kg}^{-1}\right.$ soil), temperature is in $\left({ }^{\circ} \mathrm{C}\right), \mathrm{OM}$ is in organic matter \% ( $\mathrm{g} \mathrm{OM} \mathrm{g}^{-1}$ soil). Figure $\mathrm{B} 1$ represent the topology of this ANN. Figure B2 shows the partial depence of this ANN model, compared to the mean (bagged) response.

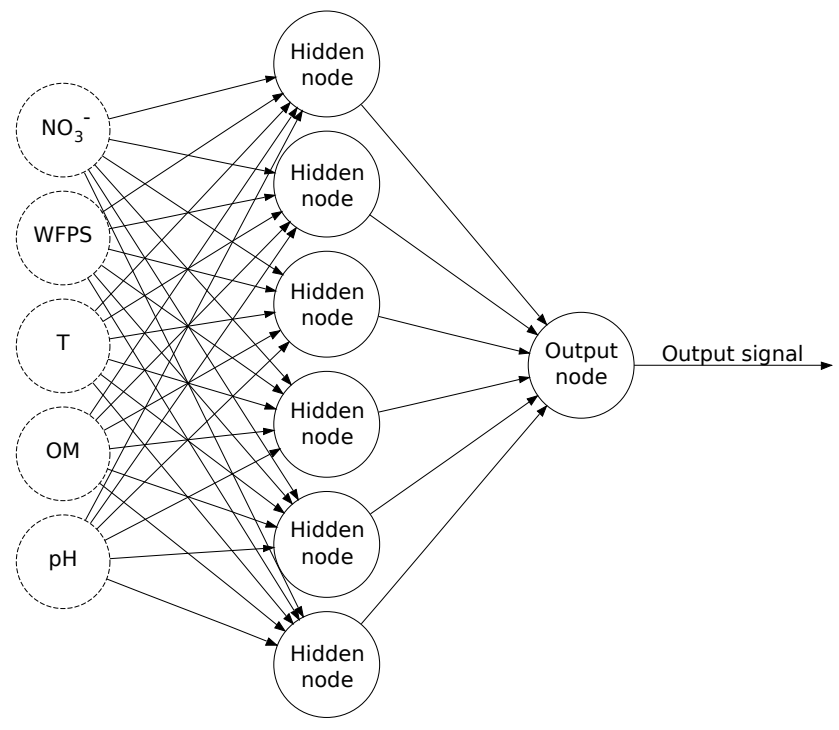

Fig. B1. ANN5(OM,pH)G model [5:6:1] topology. Solid circles represent neurons and dashed circles represent the inputs. 

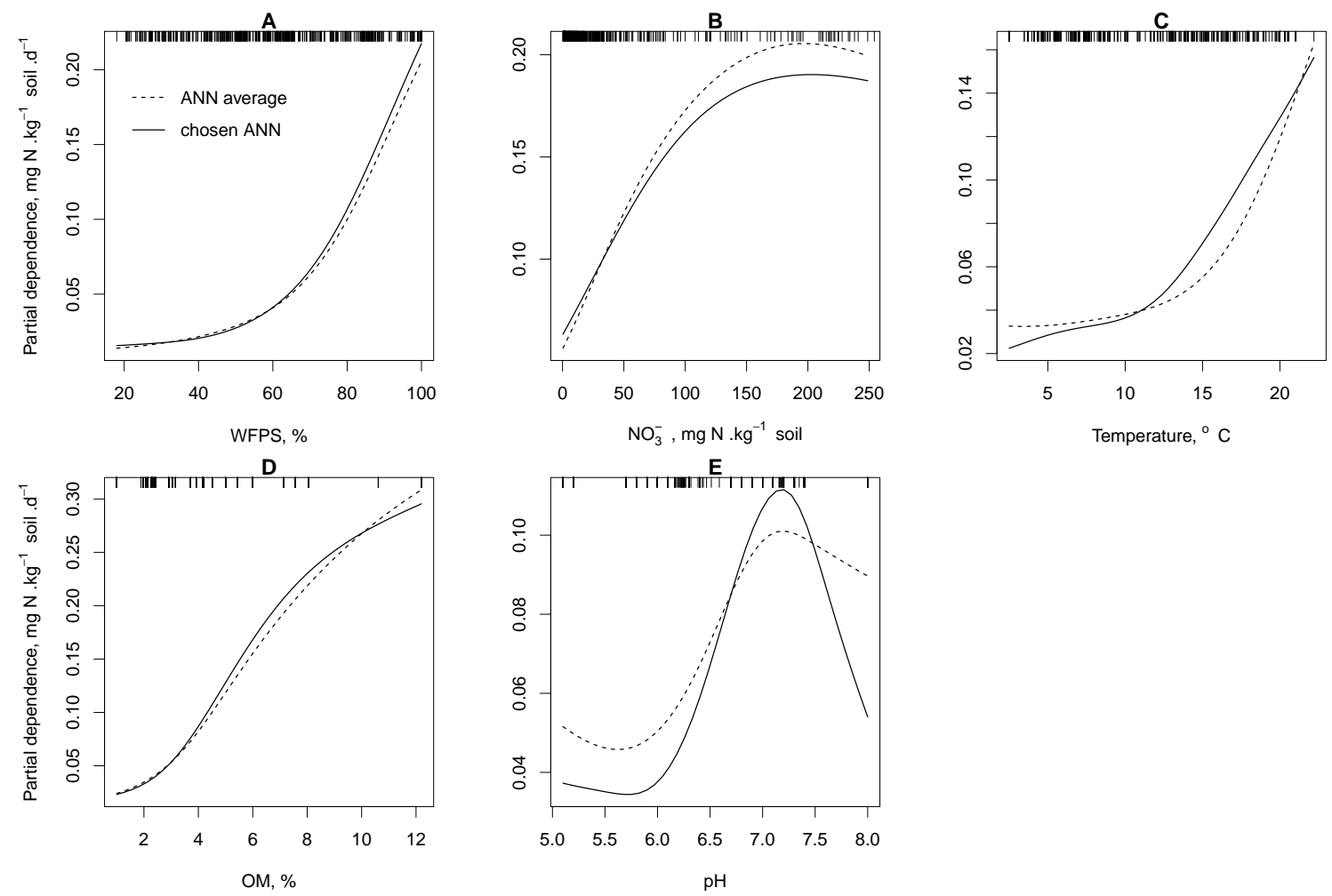

Fig. B2. Mean partial dependence of ANN5(OM,pH)G model and the partial dependence of the chosen ANN5(OM,pH)G. Rug plots on each subplot indicate the presence of the $\mathrm{x}$-axis data in the dataset (e.g. only few different values of $\mathrm{pH}$ are present in the original dataset and so few lines appear in the rug plot of subplot E).

\section{Appendix C}

\section{List of the abbreviations}

$\begin{array}{ll}\text { ANN } & \text { Artificial Neural Network } \\ \mathrm{BRT} & \text { Boosted Regression Trees } \\ \mathrm{C}_{2} \mathrm{H}_{2} & \text { acetylene } \\ \mathrm{CV} & \text { Cross-Validation } \\ \mathrm{Da} & \text { actual Denitrification } \\ \mathrm{Db} & \text { Bulk density } \\ \mathrm{DEA} & \text { Denitrifying Enzyme Activity } \\ \mathrm{LDP} & \text { long term Denitrification Potential } \\ \mathrm{ML} & \text { Machine Learning } \\ \mathrm{N} & \text { Nitrogen } \\ \mathrm{N}_{2} & \text { Di-Nitrogen } \\ \mathrm{N}_{2} \mathrm{O} & \text { Nitrous Oxyde } \\ \mathrm{NO}_{2}^{-} & \text {Nitrite } \\ \mathrm{NO}_{3}^{-} & \text {Nitrate } \\ \mathrm{NRMSE}^{-} & \text {Normalized Root Mean Squared Error } \\ \mathrm{O}_{2} & \text { Di-oxygen } \\ \mathrm{OM} & \text { Organic Matter } \\ \mathrm{PaD} & \text { Partial Derivative } \\ \mathrm{SD} & \text { Soil Depth } \\ T & \text { Temperature } \\ \text { WFPS } & \text { Water Filled Pore Space }\end{array}$

\section{Appendix D}

\section{Data point distribution in the 5 chosen factors and response data space}

Figure D1 represents the scatterplots of the combination of $\mathrm{Da}, \mathrm{NO}_{3}^{-}$, WFPS, OM, $\mathrm{pH}$ and $T$. These can be used as guidelines to evaluate the domain of validity of the ANN5(OM,pH)G model. 

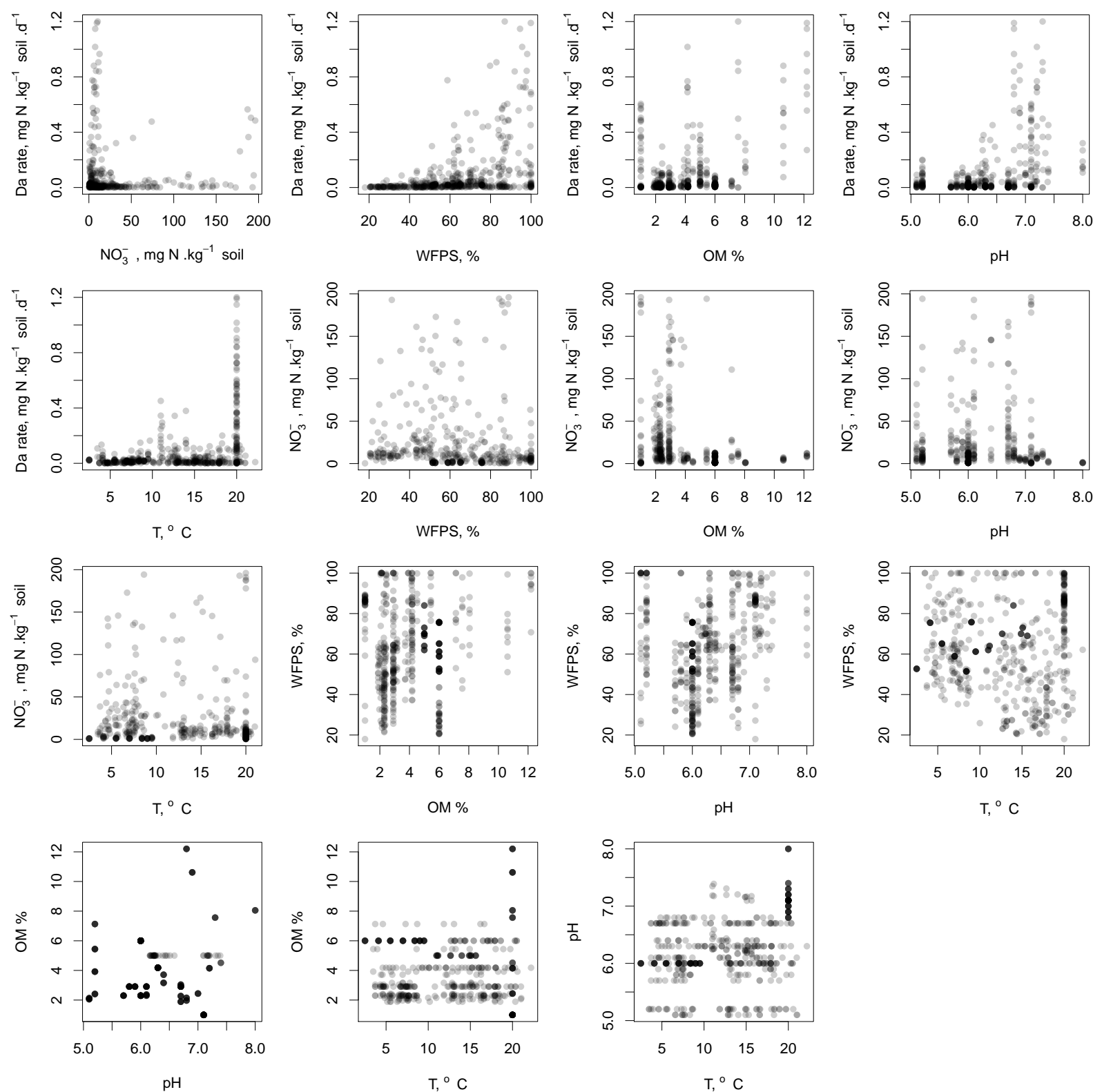

Fig. D1. Scatterplots of the combination of $\mathrm{Da}, \mathrm{NO}_{3}^{-}$, WFPS, OM, $\mathrm{pH}$ and $T$. A light grey dot represents one data point. Dots get darker as data points overlap. $\mathrm{NO}_{3}^{-}$range is limited to $200 \mathrm{mg} \mathrm{N} \mathrm{kg}^{-1}$ soil as there is few data between 200 and $800 \mathrm{mg} \mathrm{N} \mathrm{kg}^{-1}$ soil.

Acknowledgements. We would like to thank John Leathwick for his kind support regarding BRT. The work was founded by the National Institute of Water \& Atmospheric Research Capability Funds.

Edited by: E. Falge 


\section{References}

Alpaydin, E.: Introduction to Machine Learning (Adaptive Computation and Machine Learning), The MIT Press, 2004.

Arnold, J. G. and Fohrer, N.: SWAT2000: current capabilities and research opportunities in applied watershed modelling, Hydrol. Process., 19, 563-572, 2005.

Basset-Mens, C., Anibar, L., Durand, P., and van der Werf, H. M. G.: Spatialised fate factors for nitrate in catchments: Modelling approach and implication for LCA results, Sci. Total Environ., 367, 367-382, 2006.

Beaujouan, V., Durand, P., and Ruiz, L.: Modelling the effect of the spatial distribution of agricultural practices on nitrogen fluxes in rural catchments, Ecol. Model., 137, 93-105, 2001.

Beven, K.: Prophecy, Reality and Uncertainty in Distributed Hydrological Modeling, Adv. Water Resour., 16, 41-51, 1993.

Bollmann, A. and Conrad, R.: Acetylene blockage technique leads to underestimation of denitrification rates in oxic soils due to scavenging of intermediate nitric oxide, Soil Biol. Biochem., 29, 1067-1077, doi:10.1016/S0038-0717(97)00007-2, 1997.

Bousquet, O. and Elisseeff, A.: Stability and generalization, J. Mach. Learn. Res., 2, 499-526, doi:10.1162/153244302760200704, 2002.

Breiman, L.: Bagging Predictors, Machine Learning, 24, 123-140, 1996.

Breiman, L., Friedman, J., Olshen, R., and Stone, C.: Classification and Regression Trees, Wadsworth International Group, Belmont, CA, USA, 1984.

Carney, J. and Cunningham, P.: Confidence and prediction intervals for neural network ensembles, in: The International Joint Conference on Neural Networks, 1999.

Cheng, W., Tsuruta, H., Chen, G., and Yagi, K.: $\mathrm{N}_{2} \mathrm{O}$ and NO production in various Chinese agricultural soils by nitrification, Soil Biol. Biochem., 36, 953-963, 2004.

Cicerone, R.: Changes in stratospheric ozone, Science, 237, 35-42, 1987.

Cosandey, A. C., Maitre, V., and Guenat, C.: Temporal denitrification patterns in different horizons of two riparian soils, Eur. J. Soil Sci., 54, 25-37, 2003.

Cote, M., Grandjean, B. P. A., Lessard, P., and Thibault, J.: Dynamic modelling of the activated sludge process: Improving prediction using neural networks, Water Res., 29, 995-1004, 1995.

Crutzen, P. J., Mosier, A. R., Smith, K. A., and Winiwarter, W.: $\mathrm{N}_{2} \mathrm{O}$ release from agro-biofuel production negates global warming reduction by replacing fossil fuels, Atmos. Chem. Phys., 8, 389-395, doi:10.5194/acp-8-389-2008, 2008.

Cunningham, P., Carney, J., and Jacob, S.: Stability problems with artificial neural networks and the ensemble solution, Artif. Intell. Med., 20, 217-225, 2000.

Cybenko, G.: Approximation by superpositions of a sigmoidal function, Math. Control, Signal., 2(4), 303-314, 1989.

Dimopoulos, I., Chronopoulos, J., Chronopoulou-Sereli, A., and Lek, S.: Neural network models to study relationships between lead concentration in grasses and permanent urban descriptors in Athens city (Greece), Ecol. Model., 120, 157-165, doi:10.1016/ S0304-3800(99)00099-X, 1999.

Efron, B.: Better Bootstrap Confidence Intervals, J. Am. Stat. Assoc., 82, 171-185, 1987.
Elith, J., Leathwick, J. R., and Hastie, T.: A working guide to boosted regression trees, J. Anim. Ecol., 77, 802-813, 2008.

Faraggi, D. and Simon, R.: A neural network model for survival data, Stat. Med., 14, 73-82, 1995.

Firestone, M., Firestone, R., and Tiedje, J.: Nitrous oxide from soil denitrification: factors controlling its biological production, Science, 208, 749-751, 1980.

Fisher, J. and Acreman, M. C.: Wetland nutrient removal: a review of the evidence, Hydrol. Earth Syst. Sci., 8, 673-685, doi:10.5194/hess-8-673-2004, 2004.

Freund, Y. and Schapire, R. E.: A decision-theoretic generalization of on-line learning and an application to boosting, in: EuroCOLT '95: Proceedings of the Second European Conference on Computational Learning Theory, 23-37, Springer-Verlag, London, UK, 1995.

Friedman, H. and Meulman, J.: Multiple additive regression trees with application in epidemiology, Stat. Med., 22, 1365-1381, 2003.

Friedman, J. H.: Greedy Function Approximation: A Gradient Boosting Machine, Ann. Stat., 29, 1189-1232, 2001.

Gagolewski, M.: phull: p-hull: a generalization of convex hull, $\mathrm{X}-\mathrm{Y}$ hull and bounding rectangle, http://CRAN.R-project.org/ package= phull, $\mathrm{r}$ package version $0.2-1,2009$.

Gevrey, M., Dimopoulos, I., and Lek, S.: Review and comparison of methods to study the contribution of variables in artificial neural network models, Ecol. Model., 160, 249-264, 2003.

Groffman, P. M., Gold, A. J., and Jacinthe, P. A.: Nitrous oxide production in riparian zones and groundwater, Nutr. Cycl. Agroecosys., 52, 179-186, 1998.

Groffman, P. M., Altabet, M. A., Bohlke, J. K., Butterbach-Bahl, K., David, M. B., Firestone, M. K., Giblin, A. E., Kana, T. M., Nielsen, L. P., and Voytek, M. A.: Methods for measuring denitrification: Diverse approaches to a difficult problem, Ecol. Appl., 16, 2091-2122, 2006.

Haag, D. and Kaupenjohann, M.: Landscape fate of nitrate fluxes and emissions in Central Europe - A critical review of concepts, data, and models for transport and retention, Agr. Ecosyst. Environ., 86, 1-21, 2001.

Hagan, M. T., Demuth, H. B., and Beale, M.: Neural network design, PWS Publishing Company, Boston, Massachusetts, 1996.

Hansen, S., Jensen, H. E., Nielsen, N. E., and Svendsen, H.: Simulation of nitrogen dynamics and biomass production in winter wheat using the Danish simulation model DAISY, Nutr. Cycl. Agroecosys., 27, 245-259, 1991.

Heinen, M.: Application of a widely used denitrification model to Dutch data sets, Geoderma, 133, 464-473, 2006a.

Heinen, M.: Simplified denitrification models: Overview and properties, Geoderma, 133, 444-463, 2006b.

Henault, C. and Germon, J. C.: NEMIS, a predictive model of denitrification on the field scale, Eur. J. Soil Sci., 51, 257-270, 2000.

Henault, C., Bizouard, F., Laville, P., Gabrielle, B., Nicoullaud, B., Germon, J. C., and Cellier, P.: Predicting in situ soil $\mathrm{N}_{2} \mathrm{O}$ emission using NOE algorithm and soil database, Glob. Change Biol., 11, 115-127, 2005.

Hofstra, N. and Bouwman, A.: Denitrification in Agricultural Soils: Summarizing Published Data and Estimating Global Annual Rates, Nutr. Cycl. Agroecosys., 72, 267-278, 
doi:10.1007/s10705-005-3109-y, 2005.

Hornik, K., Stinchcombe, M., and White, H.: Multilayer feedforward networks are universal approximators, Neural Networks, 2, 359-366, 1989.

Hwang, S. J. and Hanaki, K.: Effects of oxygen concentration and moisture content of refuse on nitrification, denitrification and nitrous oxide production, Bioresource Technol., 71, 159-165, 2000.

IPCC: Guidelines for National Greenhouse Gas Inventories, http: //www.ipcc-nggip.iges.or.jp, access: 15 October 2010, 2006.

Irie, B. and Miyake, S.: Capabilities of three-layered perceptrons, Proceedings of the IEEE Second International Conference on Neural Networks (San Diego), 1, 641-647, 1988.

Jarvis, S. C., Hatch, D. J., and Lovell, R. D.: An improved soil core incubation method for the field measurement of denitrification and net mineralization using acetylene inhibition, Nutr. Cycl. Agroecosys., 59, 219-225, 2001.

Johnsson, H., Bergström, L., Jansson, P., and Paustian, K.: Simulated nitrogen dynamics and losses in a layered agricultural soil., Agr. Ecosyst. Environ., 18, 333-356, 1987.

Johnsson, H., Klemedtsson, L., Nilsson, A., and Svensson, B.: Simulation of field scale denitrification losses from soils under grass ley and barley, Plant Soil, 138, 287-302, 1991.

Kaufman, L. and Rousseeuw, P. J.: Finding Groups in Data: An Introduction to Cluster Analysis (Wiley Series in Probability and Statistics), Wiley-Interscience, 2005.

Knisel, W. G.: GLEAMS: Groundwater Loading Effects of Agricultural Management Systems, version 2.10, University of Georgia, Costal Plain Experiment Station, Biological and Agricultural Engineering Department, 1993.

Kralisch, S., Fink, M., Flugel, W. A., and Beckstein, C.: A neural network approach for the optimisation of watershed management, Environ. Modell. Softw., 18, 815-823, 2003.

Lehuger, S., Gabrielle, B., Oijen, M. v., Makowski, D., Germon, J. C., Morvan, T., and Hénault, C.: Bayesian calibration of the nitrous oxide emission module of an agro-ecosystem model, Agr. Ecosyst. Environ., 133, 208-222, 2009.

Leip, A., Marchi, G., Koeble, R., Kempen, M., Britz, W., and $\mathrm{Li}, \mathrm{C}$.: Linking an economic model for European agriculture with a mechanistic model to estimate nitrogen and carbon losses from arable soils in Europe, Biogeosciences, 5, 73-94, doi:10.5194/bg-5-73-2008, 2008.

Lek, S., Guiresse, M., and Giraudel, J. L.: Predicting stream nitrogen concentration from watershed features using neural networks, Water Res., 33, 3469-3478, 1999.

Li, C., Frolking, S., and Frolking, T.: A model of nitrous oxide evolution from soil driven by rainfall events: 1 . Model structure and sensitivity, J. Geophys. Res., 97, 9759-9776, 1992.

Liano, K.: Robust error measure for supervised neural network learning with outliers, IEEE T. Neural Networ., 7(1), 246-250, 1996.

Lischeid, G.: Investigating trends of hydrochemical time series of small catchments by artificial neural networks, Phys. Chem. Earth Pt. B, 26, 15-18, 2001.

Luo, J., White, R. E., Ball, P. R., and Tillman, R. W.: Measuring denitrification activity in soils under pasture: Optimizing conditions for the short-term denitrification enzyme assay and effects of soil storage on denitrification activity, Soil Biol. Biochem., 28, 409-417, 1996.
Luo, J., Tillman, R. W., and Ball, P. R.: Grazing effects on denitrification in a soil under pasture during two contrasting seasons, Soil Biol. Biochem., 31, 903-912, 1999.

Machefert, S. E., Dise, N. B., Goulding, K. W. T., and Whitehead, P.G.: Nitrous oxide emission from a range of land uses across Europe, Hydrol. Earth Syst. Sci., 6, 325-338, doi:10.5194/hess6-325-2002, 2002.

Maechler, M., Rousseeuw, P., Struyf, A., and Hubert, M.: Cluster Analysis Basics and Extensions, cluster R package, 2005.

Martin, T. L., Kaushik, N. K., Trevors, J. T., and Whiteley, H. R.: Review: Denitrification in temperate climate riparian zones, Water Air Soil Poll., 111, 171-186, 1999.

McCulloch, W. S. and Pitts, W.: A logical calculus of the ideas imminent in nervous activity, B. Math. Biophys., 5, 115-133, 1943.

Miller, M. N., Zebarth, B. J., Dandie, C. E., Burton, D. L., Goyer, C., and Trevors, J. T.: Crop residue influence on denitrification, $\mathrm{N}_{2} \mathrm{O}$ emissions and denitrifier community abundance in soil, Soil Biol. Biochem., 40, 2553-2562, 2008.

Moldrup, P., Olesen, T. R., Yoshikawa, S., Komatsu, T., and Rolston, D. E.: Predictive-descriptive models for gas and solute diffusion coefficients in variably saturated porous media coupled to pore-size distribution: II. Gas diffusivity in undisturbed soil, Soil Sci., 170, 854-866, 2005.

Molenat, J. and Gascuel-Odoux, C.: Modelling flow and nitrate transport in groundwater for the prediction of water travel times and of consequences of land use evolution on water quality, Hydrol. Process., 16, 479-492, 2002.

Mosier, A. and Kroeze, C.: Potential impact on the global atmospheric $\mathrm{N}_{2} \mathrm{O}$ budget of the increased nitrogen input required to meet future global food demands, Chemosphere - Global Change Science, 2, 465-473, 2000.

Nevison, C.: Review of the IPCC methodology for estimating nitrous oxide emissions associated with agricultural leaching and runoff, Chemosphere - Global Change Science, 2, 493-500, 2000.

Oehler, F., Bordenave, P., and Durand, P.: Variations of denitritication in a farming catchment area, Agr. Ecosyst. Environ., 120, 313-324, 2007.

Oehler, F., Durand, P., Bordenave, P., Saadi, Z., and SalmonMonviola, J.: Modelling denitrification at the catchment scale, Sci. Total Environ., 407, 1726-1737, 2009.

Olden, J. D., Joy, M. K., and Death, R. G.: An accurate comparison of methods for quantifying variable importance in artificial neural networks using simulated data, Ecol. Model., 178, 389397, 2004.

Pasti, R., de Castro, L., Coelho, G., and Von Zuben, F.: Neural network ensembles: immune-inspired approaches to the diversity of components, Nat. Comp., 9, 625-653, doi:10.1007/s11047009-9124-1, 2010.

Pernía-Espinoza, A. V., Ordieres-Meré, J. B., Martínez-de Pisón, F. J., and González-Marcos, A.: TAO-robust backpropagation learning algorithm, Neural Networks, 18, 191-204, 2005.

Perrone, M. P. and Cooper, L. N.: When Networks Disagree: Ensemble Methods for Hybrid Neural Networks, Chapman and Hall, 126-142, 1993.

Petersen, S. O., Schjonning, P., Thomsen, I. K., and Christensen, B. T.: Nitrous oxide evolution from structurally intact soil as influenced by tillage and soil water content, Soil Biol. Biochem., 
40, 967-977, 2008.

R Development Core Team: R: A Language and Environment for Statistical Computing, R Foundation for Statistical Computing, Vienna, Austria, http://www.R-project.org, ISBN 3-900051-070, 2008.

Ridgeway, G.: gbm: Generalized Boosted Regression Models, 2007.

Rousseeuw, P. J.: Silhouettes: A graphical aid to the interpretation and validation of cluster analysis, J. Comput. Appl. Math., 20, 53-65, doi:10.1016/0377-0427(87)90125-7, 1987.

Ruiz, L., Abiven, S., Durand, P., Martin, C., Vertès, F., and Beaujouan, V.: Effect on nitrate concentration in stream water of agricultural practices in small catchments in Brittany: I. Annual nitrogen budgets, Hydrol. Earth Syst. Sci., 6, 497-506, doi:10.5194/hess-6-497-2002, 2002.

Ryan, M., Müller, C., Di, H. J., and Cameron, K. C.: The use of artificial neural networks (ANNs) to simulate $\mathrm{N} 2 \mathrm{O}$ emissions from a temperate grassland ecosystem, Ecol. Model., 175, 189194, 2004.

Ryden, J., Skinner, J., and Nixon, D.: Soil core incubation system for the field measurement of denitrification using acetyleninhibition, Soil Biol. Biochem., 19, 753-757, 1987.

Ryden, J. C.: Denitrification loss from a grassland soil in the field receiving different rates of nitrogen as ammonium nitrate, Eur. J. Soil Sci., 34, 355-365, 1983.

Ryden, J. C. and Dawson, K. P.: Evaluation of the acetyleneinhibition technique for the measurement of denitrification in grassland soils, J. Sci. Food Agr., 33, 1197-1206, 1982.

Schapire, R.: The boosting approach to machine learning an overview, MSRI Workshop on Nonlinear Estimation and Classification, 2002, Springer, New York, 2003.

Sebilo, M., Billen, G., Grably, M., and Mariotti, A.: Isotopic composition of nitrate-nitrogen as a marker of riparian and benthic denitrification at the scale of the whole Seine River system, Biogeochemistry, 63, 35-51, 2003.

Seitzinger, S., Harrison, J. A., Böhlke, J. K., Bouwman, A. F., Lowrance, R., Peterson, B., Tobias, C., and Drecht, G. V.: Denitrification across landscapes and waterscapes: a synthesis, Ecol. Appl., 16, 2064-2090, doi:10.1890/1051-0761(2006) 016[2064:DALAWA]2.0.CO;2, 2006.

Simek, M. and Cooper, J. E.: The influence of soil $\mathrm{pH}$ on denitrification: progress towards the understanding of this interaction over the last 50 years, Eur. J. Soil Sci., 53, 345-354, 2002.

Simek, M., Cooper, J. E., Picek, T., and Santruckova, H.: Denitrification in arable soils in relation to their physicochemical properties and fertilization practice, Soil Biol. Biochem., 32, 101-110, 2000.

Simek, M., Jisova, L., and Hopkins, D. W.: What is the so-called optimum $\mathrm{pH}$ for denitrification in soil?, Soil Biol. Biochem., 34, 1227-1234, 2002.

Smith, M. and Tiedje, J.: Phases of denitrification following oxygen depletion in soil, Soil Biol. Biochem., 11, 261-167, 1979.
Smits, J. R. M., Breedveld, L. W., Derksen, M. W. J., Kateman, G., Balfoort, H. W., Snoek, J., and Hofstraat, J. W.: Pattern classification with artificial neural networks: classification of algae, based upon flow cytometer data, Anal. Chim. Acta, 258, 11-25, 1992.

Sogbedi, J., van Es, H., and Huton, J.: N fate and transport under variable cropping history and fertilizer rate on loamy sand and clay loam soils: I. Calibration of the LEACHM model, Plant Soil, 229, 57-70, 2001.

Suen, J. P. and Eheart, J. W.: Evaluation of neural networks for modeling nitrate concentrations in rivers, J. Water Res. Pl.-Asce, 129, 505-510, 2003.

Telszewski, M., Chazottes, A., Schuster, U., Watson, A. J., Moulin, C., Bakker, D. C. E., González-Dávila, M., Johannessen, T., Körtzinger, A., Lüger, H., Olsen, A., Omar, A., Padin, X. A., Ríos, A. F., Steinhoff, T., Santana-Casiano, M., Wallace, D. W. R., and Wanninkhof, R.: Estimating the monthly $p \mathrm{CO}_{2}$ distribution in the North Atlantic using a self-organizing neural network, Biogeosciences, 6, 1405-1421, doi:10.5194/bg6-1405-2009, 2009.

Tibshirani, R.: A Comparison of Some Error Estimates for Neural Network Models, Neural Comput., 8, 152-163, doi:10.1162/ neco.1996.8.1.152, 1996.

Tiedje, J.: Methods of Soil Analysis (2nd Edn.), chap. Denitrification, Madison, 1011-1026, 1982.

Tiedje, J. M., Simkins, S., and Groffman, P. M.: Perspectives on measurement of denitrification in the field including recommended protocols for acetylene based methods, Plant Soil, 115, 261-284, 1989.

Turpin, N., Bontems, P., Rotillon, G., Bärlund, I., Kaljonen, M., Tattari, S., Feichtinger, F., Strauss, P., Haverkamp, R., Garnier, M., Porto, A. L., Benigni, G., Leone, A., Ripa, M. N., Eklo, O.-M., Romstad, E., Bioteau, T., Birgand, F., Bordenave, P., Laplana, R., Lescot, J.-M., Piet, L., and Zahm, F.: AgriBMPWater: systems approach to environmentally acceptable farming, Policies and Tools for Sustainable Water Management in the European Union, Environ. Modell. Softw., 20, 187-196, 2005.

Vinten, A. J. A., Castle, K., and Arah, J. R. M.: Field evaluation of models of denitrification linked to nitrate leaching for aggregated soil, Eur. J. Soil Sci., 47, 305-317, 1996.

Yoshinari, T. and Knowles, R.: Acetylene inhibition of nitrous oxide reduction by denitrifying bacteria, Biochem. Biophy. Res. Co., 69, 705-710, 1976.

Yoshinari, T., Hynes, R., and Knowles, R.: Acetylene inhibition of nitrous oxyde reduction and measurement of denitrification and nitrogen fixation in soil, Soil Biol. Biochem., 9, 177-183, 1977.

Zaman, M. and Nguyen, M.: Effect of lime or zeolite on $\mathrm{N}_{2} \mathrm{O}$ and $\mathrm{N}_{2}$ emissions from a pastoral soil treated with urine or nitrate$\mathrm{N}$ fertilizer under field conditions, Agr. Ecosyst. Environ., 136, 254-261, doi:doi:10.1016/j.agee.2009.12.002, 2010. 